\title{
Physical and Mathematical Modelling of Steelmaking Tundish Operations: A Review of the Last Decade (1999-2009)
}

\author{
Kinnor CHATTOPADHYAY, Mihaiela ISAC and Roderick I. L. GUTHRIE \\ McGill Metals Processing Centre, Department of Mining and Materials Engineering, McGill University, Montreal, Quebec, H3A \\ 2B2 Canada. E-mail: roderick.guthrie@mcgill.ca
}

(Received on September 24, 2009; accepted on November 24, 2009)

\begin{abstract}
A great number of research articles on the physical and mathematical modelling of tundish operation have been reported in the literature. Mazumdar and Guthrie ${ }^{1)}$ summarized a large portion of these in a review article in ISIJ published in 1999. Because of the growing importance of tundish metallurgy and stringent demands regarding steel quality, a lot of further work has been done on tundish technology from1999 till date. This review summarizes the basics of physical modelling, mathematical modelling and the work of different researchers around the globe in the last decade i.e. 1999 to 2009. Current trends in research have been reviewed and critiqued.
\end{abstract}

KEY WORDS: continuous casting; tundish; physical modelling; mathematical modelling.

\section{Introduction}

In the continuous casting of steel, the tundish is basically an intermediate vessel placed between the ladle and the mould so as to distribute and supply liquid steel to different moulds at an approximately constant rate. In recent years, with continuing emphasis on superior steel quality, the tundish has become more of a continuous reactor than merely a distribution vessel. Thus a modern day steel making tundish is designed to provide maximum opportunity for carrying out various metallurgical operations such as inclusion separation, inclusion floatation, alloy trimming of steel, and thermal and chemical homogenization. All these have led to the development of a separate area of secondary steelmaking referred to as "tundish metallurgy". 1) Thus significant efforts have been made by researchers around the globe in the last decade, i.e., 1999 to 2008 to fully exploit and enhance the potential of continuous casting tundish as a molten steel refining vessel. Research activities concerning the associated theoretical and applied aspects have naturally led to a large number of publications. Mazumdar and Guthrie $^{1)}$ in 1999 concluded that numerous experimental and theoretical studies have been carried out using both aqueous models and industrial units to investigate various transport phenomena of relevance to continuous casting tundish system. A wide range of tundish geometries along with numerous designs of flow modifiers were applied to investigate primarily the floatation of inclusions from tundishes as a function of operating variables. In addition to these, the role of increased throughput rate, electromagnetic stirring and auxiliary heating on tundish performance were also investigated. Flow conditions suitable to facilitate inclusion separation by floatation can be created by insertion of flow modifiers. However, the optimal design of such flow modifiers and their location within the tundish is highly dependent on the tundish geometry, operating conditions and size range of inclusions present within the molten steel. Mixing, both thermal and material, on the other hand require significantly different flow conditions. Useful inferences on industrial tundish performance can be made from observations derived from reduced scale water models. Extensive mathematical modelling of fluid flow and transport phenomena and the concurrent validation of mathematical model predictions against laboratory, as well as plant scale experimental data, indicate that a reasonably accurate mathematical framework now exists for effective tundish design and process analysis. For simplicity and lucidity the whole review has been divided into three parts i.e. Physical modelling, Mathematical modelling and Work of different researchers.

\section{Physical Modelling}

Physical modelling involves the use of a low temperature aqueous analogue, generally water, to represent molten metal in the tundish. Water flow in a transparent model tundish can be used to observe the melt flow physically. Models can be either reduced scale or full scale. There are certain advantages to using a full scale model over a reduced scale model and these are mentioned by Guthrie and Isac. ${ }^{2)}$ For a faithful representation of flow in the model tundish, there should be constant ratios between corresponding quantities in the model and the actual tundish. For melt flow in tundishes, the states of similarity normally include geometric, kinematic, dynamic and thermal similarities. Here, the industrial vessel is known as the prototype, and its laboratory-scale counterpart is known as the model. Laboratory-scale modelling of various secondary steel- 
making operations has most frequently used water as the modelling medium to represent molten steel. The most important single property in this context, apart from its ubiquity, is that its kinematic viscosity (that is, molecular viscosity/density) is essentially equivalent to that of molten steel at $1600^{\circ} \mathrm{C}$ (i.e., within $10 \%$ ). Flow visualization experiments in aqueous systems using dyes or other tracers have therefore proved to be very helpful in developing a qualitative understanding of various flows in real liquid steel systems. Similarly, more detailed information on flow characteristics has also been possible by measuring velocity fields by tracking the motion of neutrally buoyant particles or by using hot wire or hot film anemometry, or by laser Doppler anemometry, and, lately, by PIV (Particle Image Velocimetry). In addition, measurements of residence time distribution to characterize mixing in water model experiments using dye, acids, or $\mathrm{KCl}$ salt solution have proved very popular and efficient. Having realized the advantages of using water as the representative fluid, it is now appropriate to discuss the general problem of how to physically model or characterize metallurgical processes. It is important to note here that, if the same forms of dimensionless differential equations and boundary conditions apply to two or more such metallurgical operations, and if an equivalence of dimensionless velocity, temperature, pressure or concentration fields, etc. also exist between the two, then one of them becomes a faithful representation of the other; i.e., one can be termed as a model of the other. This is a general statement of the need for similarity between a model and a prototype, which requires that there be constant ratios between corresponding quantities. The state of similarity between a model and a full-scale system includes geometric, mechanical, thermal, and chemical similarity. Mechanical similarity is further subdivided into static, kinematic, and dynamic similarity. However, in modelling of these steelmaking operations, static similarity has no relevance. The various states of similarity are discussed in standard texts in great detail. ${ }^{3)}$

Recently a new dimensionless number has gained wide importance and that is the Bond number. The Bond number, notated Bo, is a dimensionless number expressing the ratio of body forces (often gravitational or buoyancy) to surface tension forces

$$
\mathrm{Bo}=\frac{\rho g L^{2}}{\sigma}
$$

The Bond number is a measure of the importance of surface tension forces compared to body forces. A high Bond number indicates that the system is relatively unaffected by surface tension effects; a low number (typically less than one is the requirement) indicates that surface tension dominates. Intermediate numbers indicate a non-trivial balance between the two effects. The Bond number is the most common comparison of gravity and surface tension effects and it may be derived in a number of ways, such as scaling the pressure of a drop of liquid on a solid surface. It is usually important, however, to find the right length scale specific to a problem by doing a ground-up scale analysis. Other dimensionless numbers that are related to the Bond number ${ }^{4)}$ are,

$$
\mathrm{Bo}=\mathrm{Eo}=2 \mathrm{Go}^{2}=\mathrm{De}^{2}
$$

where Eo, Go, and De are respectively the Eötvös, Goucher, and Deryagin numbers. The "difference" between the Goucher and Deryagin numbers is that the Goucher number (arises in wire coating problems) uses the letter $R$ to represent length scales while the Deryagin number (arises in plate film thickness problems) uses $L$.

The We and Bo similarity become very important when we simulate the slag phase with oils or emulsions. Different liquids like Benzene, Toluene, $\mathrm{CCl}_{4}$, oils, paraffin oil ${ }^{5)}$ etc. have been used to simulate the slag phase but G. A. Irons ${ }^{6}$ ) noted that paraffin oil is the best liquid for simulating the slag phase in water models.

If we use reduced scale models we cannot simultaneously achieve Reynolds number similarity and Froude number similarity as only one of them can be respected. Since the flows in the tundish are associated with high Reynolds numbers, inertial forces far exceed laminar viscous forces and correspond to the Newtonian range which are insensitive to Re. By contrast inertial to gravitational forces are similar in magnitude. As such, flows in the tundish are Froude dominated and hence Froude number similarity is maintained between the model and the prototype. However in full scale modelling both Re and Fr similarities can be achieved simultaneously.

The above mentioned similarity criteria are alright when there are no temperature changes and no effect of buoyancy forces. However, for non isothermal modelling we should use a form of modified Froude number, representing the inverse ratio of inertial forces to differences in buoyancy forces.

Damle and Sahai ${ }^{7)}$ called this the Tundish Richardson number $(\mathrm{Tu})$. Tu denotes the ratio of buoyancy force to the inertial force and is expressed as

$$
\mathrm{Tu}=\frac{\mathrm{Gr}}{\mathrm{Re}^{2}}=\frac{g L \beta \Delta T_{0}}{U^{2}}
$$

If inclusion removal modelling is done then

$$
\frac{V_{f, m}}{V_{f, p}}=\frac{V_{r, m}}{V_{r, p}}
$$

is to be maintained where $V_{f}$ and $V_{r}$ are fluid and Stokes rise velocities respectively. ${ }^{8}$ However, the inclusions rise velocities may not always be in the Stoke's regime.

In modelling heat transfer operations, thermally similar systems are those in which corresponding temperature differences bear a constant ratio to one another at corresponding positions. When the systems are moving, kinematic similarity is a prerequisite to any thermal similarity. Thus, the heat transfer ratio by conduction, convection, and/or radiation to a certain location in the model must bear a fixed ratio to the corresponding rates in the prototype. ${ }^{9)}$ Finally, for chemical similarity between a model and a full scale system, the dynamic and thermal similarity first must be satisfied. The former, since mass transfer and chemical reaction usually occur by convective and diffusive processes during motion of reacting material through the system, and the latter since chemical kinetics are normally temperature 
dependent.

\section{Mathematical Modelling}

\subsection{Introduction}

Mathematical modelling is an alternative approach for visualising flow fields inside a tundish. In mathematical modelling, the turbulent Navier-Stokes equation is solved in a boundary fitted coordinate system to predict the velocity distributions. Analytical solution of 3D Navier-Stokes equation is not possible and so we have to go for numerical methods and for numerical solution of these equations a lot of commercial software packages like FLUENT, CFX, FLOW-3D, PHOENICS, FIDAP, COMSOL etc. have been marketed, and have allowed CFD to become an increasingly common tool for the non-experts. Various turbulence models are available like the $\kappa-\varepsilon$, RNG $\kappa-\varepsilon$, Realizable $\kappa-\varepsilon$, etc. Extensive review of the published literature shows that the basic framework of the mathematical modelling used in tundish research can be divided into three sub divisions, namely (a) defining the problem, which is done by expressing the process in terms of some physical variables using partial differential equations, with appropriate operating and boundary conditions, then (b) 'grid generation of domain and discretization' of those partial differential equations into algebraic form, using different schemes and the third part (c) is, 'solution to those discretized equations' using numerical techniques. The main physical variable related to any flow field is the velocity. Since other properties follow directly from the velocity field, determination of the velocity is the prime step in solving a flow problem. For this reason, velocity was chosen as the primary physical variable for all mathematical modelling studies. Realizing the fact that liquid steel flows within a tundish is three dimensional and turbulent in nature, almost all of the mathematical modelling works published in literature in the recent past, assume the flows to be three dimensional and turbulent. As the equation of continuity and equation of "Conservation of momentum" describes the fluid flow in mathematical terms, they are, along with the treatment of turbulence and boundary conditions, used as the starting point of all mathematical modelling studies for tundishes. In addition to using the above equations different researchers used different equations, to model different parameters. Some included an energy equation to predict temperature distribution under non isothermal conditions. ${ }^{10-12)}$ Other researchers included some other differential equations to describe inclusion trajectory and inclusion number density distributions. ${ }^{24,25)}$ Modelling has also been used to predict parameters like 'Residence Time Distribution'12) (RTD), the distribution of top surface slag layer, ${ }^{24)}$ etc. It is practically impossible to solve these equations numerically by Direct Numerical Simulation (DNS) because of its requirement of unrealistically high computational memory, power and time. So for most of the CFD problems, rather than solving the turbulent Navier-Stokes equation in its original form, turbulence modelling is incorporated to capture the critical effects of turbulent flow without having to resolve the actual small length and time scales of turbulent motion. One of the most popular practices is of averaging the 'Continuity' and 'Navier-Stokes' equations first and then devis- ing means for solving the resulting system of equations for mean quantities of velocity and pressure. The approach along this line was first introduced by Reynolds and is called as Reynolds Averaged Navier-Stokes (RANS) equation. Here the equations are averaged over a time scale, which is long compared with the time scale of the turbulent motion but small compared with the unsteady mean flow. In this approach instantaneous velocity and pressure are decomposed into the mean and fluctuating part and incorporated in continuity and Navier-Stokes equations.

$$
\begin{gathered}
\frac{\partial \bar{U}_{i}}{\partial x_{i}}=0 \ldots \ldots \ldots \ldots \ldots \ldots \ldots \ldots \ldots \ldots \ldots \ldots \ldots \ldots \ldots \ldots \ldots \\
\frac{\partial \bar{U}_{i}}{\partial t}+U_{j} \frac{\partial \bar{U}_{i}}{\partial x_{j}}=-\frac{1}{\rho} \frac{\partial \bar{P}}{\partial x_{i}}+v \nabla^{2} \bar{U}_{i}-\frac{\partial\left(\overline{u_{i}^{\prime} u_{j}^{\prime}}\right)}{\partial x_{j}}
\end{gathered}
$$

In Eq. (3.2) the term $\overline{u_{i}^{\prime} u_{j}^{\prime}}$ is called the 'Reynolds stress tensor' $R_{i j}$. However Eqs. (3.1) and (3.2) do not form a closed system of equations. The mean pressure and velocity can only be calculated if the six unknown components of the Reynolds stress tensor are expressed in terms of mean velocity field in a consistent way. The most popular turbulence modelling comprises the various strategies that were developed to close one or more of the RANS equations. These strategies usually aim only towards engineering accuracy.

\subsection{Turbulence Models}

Among different methods, the most widely used concepts in the present day turbulence models for practical engineering applications is the "Eddy viscosity" concept. In analogy to the viscous stresses in laminar flows, Boussinesq suggested that the turbulent stresses are proportional to the mean-gradients of velocity. In this concept the 'Reynolds stress tensor' $R_{i j}$, is expressed as Eq. (3.3).

$$
R_{i j}=-v_{t}\left(\frac{\partial \overline{U_{i}}}{\partial x_{j}}+\frac{\partial \overline{U_{j}}}{\partial x_{i}}\right)+\frac{2}{3} \kappa \delta_{i j}
$$

Eddy viscosity $v_{t}$ has units of length multiplied by velocity and can be expressed as

$$
v_{t} \propto v_{0} l_{0} \quad \text { or } \quad v_{t} \propto l_{0}^{2} / \tau_{0}
$$

The models adopting the eddy viscosity concept are distinguished according to how they plan to estimate $v_{t}$. So the RANS equations in the eddy viscosity model become:

$$
\begin{aligned}
\frac{\partial \overline{U_{i}}}{\partial t}+\overline{U_{j}} \frac{\partial \overline{U_{i}}}{\partial x_{j}}= & -\frac{\partial}{\partial x_{i}}\left(\frac{\bar{P}}{\rho}+\frac{2}{3} \kappa \delta_{i j}\right)+v \nabla^{2} \overline{U_{i}} \\
& +\frac{\partial}{\partial x_{j}}\left[v_{i}\left(\frac{\partial \overline{U_{i}}}{\partial x_{j}}+\frac{\partial \overline{U_{j}}}{\partial x_{i}}\right)\right] \ldots \ldots
\end{aligned}
$$

Of the different turbulent models available in literature, only a few were used in tundish modelling. Prandtl's 'Mixing Length Model', is one of the early turbulence models 
used in tundish modelling. In this model Prandtl defined the mixing length, $l_{m}$, as the distance travelled by a fluid lump in the transverse direction before the mean velocity changes by an amount equal to the transverse fluctuation velocity. He also postulated that: the turbulence length scale $l_{0}$, is equal to the mixing length $l_{m}$, and velocity scale $v_{0}$, is equal to the mean velocity gradient times the mixing length. Accordingly Eq. (3.4) becomes:

$$
v_{t}=l_{m}^{2}\left|\frac{d \bar{U}}{d y}\right|
$$

This model is closed when mixing length is specified. In very early modelling attempt, Debroy and Sychterz, ${ }^{13)}$ had used this turbulence model for their numerical studies of flow pattern in a tundish and specified mixing length $l_{m}=0.4 d$ where $d$ is the distance from the nearest wall. But later investigations had shown that the mixing length, $l_{m}$, which according to the Prandtl's postulate equals to the turbulent length scale, varies within the turbulent boundary layer. So assignment of a single value for $l_{m}$ throughout the flow field is questionable and is one of the weaknesses of this turbulent model. Though this model was not used in any further tundish modelling approach in later years, the 'Law of the Wall', which is used for boundary conditions for more sophisticated turbulence models, was used frequently.

To date the most popular approach using eddy viscosity models are the two equation models. In these models two separate transport equations are solved to determine the length and velocity scales in Eq. (3.4) for eddy viscosity $v_{t}$. There are many types of two equation models. Out of them, $\kappa-\varepsilon$ models are most popular.

A review of the literature on mathematical modelling in tundishes reveals that most of the researchers used the standard $\kappa-\varepsilon$ model of Launder and Spalding ${ }^{14)}$ to calculate eddy viscosity. In this model the length and the time scales are built up from the turbulent kinetic energy, $\kappa$ and the turbulent dissipation rate, $\varepsilon$, using the dimensional arguments

$$
l_{0} \propto \frac{\kappa \sqrt{\kappa}}{\varepsilon} \text { and } \tau_{0} \propto \frac{\kappa}{\varepsilon}
$$

Equations (3.4) and (3.7) yields

$$
v_{t}=C_{\mu} \frac{\kappa^{2}}{\varepsilon}
$$

where $C_{\mu}$ is an empirical constant.

The turbulent kinetic energy $(\kappa)$ and the dissipation rate $(\varepsilon)$ are obtained by solving the modelled transport Eqs. (3.9) and (3.10), respectively.

$$
\begin{aligned}
\frac{\partial \kappa}{\partial t}+\overline{U_{j}} \frac{\partial \kappa}{\partial x_{j}}= & v_{t} \frac{\partial \overline{U_{i}}}{\partial x_{j}}\left(\frac{\partial \overline{U_{i}}}{\partial x_{j}}+\frac{\partial \overline{U_{j}}}{\partial x_{i}}\right) \\
& -\varepsilon+\frac{\partial}{\partial x_{i}}\left[\left(v+\frac{v_{t}}{\sigma_{\kappa}}\right) \frac{\partial \kappa}{\partial x_{i}}\right]
\end{aligned}
$$

$$
\begin{aligned}
\frac{\partial \varepsilon}{\partial t}+\overline{U_{j}} \frac{\partial \varepsilon}{\partial x_{j}}= & C_{\varepsilon 1} \frac{\varepsilon}{\kappa} v_{t} \frac{\partial \overline{U i}}{\partial x_{j}}\left(\frac{\partial \overline{U_{i}}}{\partial x_{j}}+\frac{\partial \overline{U_{j}}}{\partial x_{i}}\right) \\
& -C_{\varepsilon 2} \frac{\varepsilon^{2}}{\kappa}+\frac{\partial}{\partial x_{i}}\left[\left(v+\frac{v_{t}}{\sigma_{\varepsilon}}\right) \frac{\partial \varepsilon}{\partial x_{i}}\right]
\end{aligned}
$$

Other researchers used another $\kappa-\varepsilon$ model, which is derived from the instantaneous Navier-Stokes equation, using a mathematical technique called "Renormalization Group" (RNG) methods. This, RNG $\kappa-\varepsilon$ model results in some different constants with different values. Equations (3.11) and (3.12) are the model equations for $\kappa$ and $\varepsilon$ respectively, used in this model.

$$
\begin{array}{r}
\frac{\partial \kappa}{\partial t}+\overline{U_{i}} \frac{\partial \kappa}{\partial x_{i}}=v_{t} S^{2}-\varepsilon+\frac{\partial}{\partial x_{i}}\left(\alpha v_{t} \frac{\partial \kappa}{\partial x_{i}}\right) \ldots(3.11) \\
\frac{\partial \varepsilon}{\partial t}+\overline{U_{i}} \frac{\partial \varepsilon}{\partial x_{i}}=C_{\varepsilon 1} \frac{\varepsilon}{\kappa} v_{t} S^{2}-C_{\varepsilon 2} \frac{\varepsilon^{2}}{\kappa}-R+\frac{\partial}{\partial x_{i}}\left(\alpha v_{t} \frac{\partial \varepsilon}{\partial x_{i}}\right)
\end{array}
$$

Here $S$ is the modulus of the mean rate of strain tensor, defined as $S^{2}=2 S_{i j} S_{i j}$. Comparing with $\varepsilon$ equation of standard $\kappa-\varepsilon$ model, one extra term $R$ arises in this model. It is expressed as:

$$
R=\frac{C_{\mu} \eta^{3}\left(1-\eta / \eta_{0}\right)}{1+\beta \eta^{3}} \frac{\varepsilon^{2}}{\kappa}
$$

For turbulent viscosity calculations, this model uses the relation: $v_{t}=0.084\left(\kappa^{2} / \varepsilon\right)$. The constant 0.084 could be treated as $C_{\mu}$, as in the standard $\kappa-\varepsilon$ model with a different value.

The values of the different constants appearing in the standard $\kappa-\varepsilon$ model and $\mathrm{RNG} \kappa-\varepsilon$ model are reported in literature.

To evaluate the effect of different turbulence models on the prediction of different parameters, many researchers have carried out comparative studies. Schwarze et al. ${ }^{54,64)}$ used different turbulence models in their prediction of flow field and dispersed phase behavior. Though their predicted velocity field using standard $\kappa-\varepsilon$ and RNG $\kappa-\varepsilon$ models do not seem to vary much, the mean turbulent quantities differ significantly. Comparing the results with the available experimental data, they concluded, that the RNG model approximates the turbulence in flow situations with a high curvature of streamlines better than other models. Though the literature survey shows, that most of the simulation works were done by using the standard $\kappa-\varepsilon$ turbulence model, they ${ }^{15)}$ argued that this model, over predicts $\kappa$ values, because it does not take into account the fact that the strain rate of the flow field influences turbulence.

Hou and $\mathrm{Zou}^{15)}$ reported the same type of conclusion. They compared the standard $\kappa-\varepsilon$ turbulence model with the RNG $\kappa-\varepsilon$ turbulence model, while numerically simulating a swirling flow tundish (already mentioned earlier). They also obtained convergent result more easily using the RNG $\kappa-\varepsilon$ turbulence model and concluded that this model is 
more appropriate for swirling motion. By far the most elaborate computational study on tundish performance prediction was done by Jha et al. ${ }^{16)}$ They studied the effect of different turbulence models on residence time distribution prediction. Apart from these, in the authors knowledge, they were the only researchers who applied LES (Large eddy simulation) in tundish modelling. The models like standard $\kappa-\varepsilon$, RNG $\kappa-\varepsilon$, Chen-Kim $\kappa-\varepsilon$, LES etc. predicts gross flow properties fairly well, while others like LamBremhorst low Reynolds number $\kappa-\varepsilon$ model predicts initial variation better than the others.

More recently some researchers ${ }^{17-19)}$ used the 'Realizable $\kappa-\varepsilon$ model' of Shih et al. ${ }^{20)}$ This is basically a new eddy viscosity model, which consists of a new model dissipation rate equation and a new eddy viscosity formulation. The $\varepsilon$ model equation and the eddy viscosity formulation are given by Eqs. (3.13) and (3.14) respectively.

$$
\begin{aligned}
\frac{\partial \varepsilon}{\partial t}+\overline{U_{j}} \frac{\partial \varepsilon}{\partial x_{j}}= & C_{\varepsilon 1} \frac{\varepsilon}{\kappa} C_{3 \varepsilon} \beta g_{i} \frac{v}{\operatorname{Pr}} \frac{\partial T}{\partial x_{j}}+C_{1} S \varepsilon \\
& -C_{2} \frac{\varepsilon^{2}}{\kappa+\sqrt{v \varepsilon}}+\frac{\partial}{\partial x_{j}}\left[\left(v+\frac{v_{t}}{\sigma_{\varepsilon}}\right) \frac{\partial \varepsilon}{\partial x_{j}}\right]
\end{aligned}
$$

$$
v_{t}=C_{\mu} \frac{\kappa^{2}}{\varepsilon}
$$

Unlike in the standard $\kappa-\varepsilon$ or RNG $\kappa-\varepsilon$ models, $C_{\mu}$ is not a constant, but is defined as shown below in Eq. (3.15).

$$
C_{\mu}=\frac{1}{A_{0}+A_{s} \frac{\kappa U^{*}}{\varepsilon}}
$$

where $A_{0}$ and $A_{s}$ are model constants and $U^{*}$ values are to be calculated in terms of the mean rate of rotation and mean rate of strain tensor.

The most widely used turbulence model is the standard $\kappa-\varepsilon$. In general, as stated earlier, the standard $\kappa-\varepsilon$ model tends to overestimate mixing situations where highly turbulent and essentially laminar regions coexist, such as in tundish. To adequately predict such situations the model must be able to represent the existence probability of the turbulent fluid at a particular location. Realizing this fact, Ilegbusi ${ }^{21)}$ used a two fluid model of turbulence to predict the flow behaviour in a tundish. This model essentially considers the system to be composed of two interpenetrating fluids [Turbulent and Non turbulent fluid]. They are allowed to exchange mass, momentum and energy at the interface. At any location, transport equations would be solved for the characteristics of each fluid, including velocity components, temperature, and volume fractions. The volume fraction of the turbulent fluid provides a measure of the intermittency or turbulence. The model is therefore well suited to represent the whole tundish domain, the intermittency factor being high in the turbulent inlet region and low in the quiescent region.

In wall-bounded turbulent flow, like in a tundish, the presence of solid walls has a strong effect on flow charac- teristics. So all the researchers who tried to model the flows within the tundish, had to use special treatments like the wall function approach or the low Reynolds number model approach to tackle the boundaries. However, when there are strong re-attaching and separating flows, the wall function approach is not recommended.

Only a very few researchers ${ }^{22,23)}$ have used the Low Reynolds number method. In this approach, computations are carried out all the way to the walls and hence a very fine grid is required near the wall. Some researchers ${ }^{23)}$ used the low Reynolds number model of Launder and Jones to study the residence time distribution in a 6-strand tundish, and found better agreement with experimental result. While the low Reynolds number models like the model of Lam and Bremhorst [used by Refs. 22, 23)] and Chen-Kim low Reynolds number (with and without Yap correction) [used by Ref. 16)] were used only for comparison purpose.

Apart from the above mentioned models, some other turbulence models like Length velocity algebraic model (LVEL) [used by Refs. 24, 25)], $\kappa-\omega$ turbulence model [used by Ref. 27)], Chen-Kim (CK) [used by Ref. 61)], and more recently Reynolds Stress model (RSM) [used by Refs. 19, 26-28)] were also used. A discussion of all would require a separate review.

The capabilities of RANS models are limited. Under certain conditions these models can be very accurate, but these are not suitable for transient flows, because the averaging process wipes out most of the important characteristics of a time-dependent solution. On the other hand, 'Direct Numerical Simulation' (DNS), which is a direct solution of turbulent Navier-Stokes equation, is not practicable for 99.9\% of CFD problems, because of its requirement of unrealistically high computational power and time. As a result a new simulation technique, called 'Large eddy simulation' (LES) also became very popular. In LES, the contribution of the large scale eddies to the momentum and energy transfer is computed exactly as DNS, while the effect of the small eddies are modeled. The distinction between the large and small eddies is done by filtering operation. But exhaustive survey of literature shows only Jha et al. ${ }^{16)}$ applied LES technique during modelling of tundish.

\subsection{Solution to the Set of PDE's}

Due to the complex nature of the governing equations and geometries of the tundishes, analytical solutions for the equations are not possible in a practical sense. So different numerical techniques are devised to solve these transport equations. The numerical solution aims to provide the values of the variables at some discrete number of points in the domain of interest. These points are called grid points, nodes or cell centroids, depending on the scheme it follows. The conversion of differential equations into a set of discrete algebraic equations requires the discretization of the domain. This is called mesh generation or meshing.

The different techniques are:

1. Finite Difference Method (FDM)

2. Finite Element Method (FEM)

3. Spectral Methods

4. Hybrid Schemes (like Control Volume Finite Element Method, Control volume Finite Difference Method etc.) 
All the techniques perform three basic steps. Namely:

(i) Approximation of unknown flow variables by means of simple functions

(ii) Discretization by substitution of the approximations into the governing flow equations

(iii) Subsequent mathematical solutions

Close examination of the three components of the momentum equation and continuity equation shows that they are closely coupled, because all velocity components appear in each momentum and continuity equation. The most complex issue is to know the pressure term in the momentum equations. Because there is no equation available for pressure for the case of incompressible fluid flows like the flow in a tundish. These problems are generally tackled by the use of different iterative solution strategies like SIMPLE, SIMPLER, SIMPLEC, PISO $^{29,30)}$ etc.

\section{Research in the Last Decade}

After giving a brief description of physical modelling and mathematical modelling a comprehensive review of the work of different researchers in the last decade has been done hereafter in ascending order of chronology. Fan and Hwang, ${ }^{31)}$ in 2000, developed a mathematical model to analyze the fluid flow phenomena of liquid steel in the tundish, during its filling stage and subsequent casting operation in the continuous casting process of steel. The ultimate goal was to ensure smooth initial casting operations without nozzle clogging. The mathematical model was developed using a CFD technique, named SOLA-MAC and the famous $\kappa-\varepsilon$ turbulence model. SOLA-MAC (Solution algorithm marker and cell) has the capability to handle transient flow problem with highly distorted free surfaces. The SOLA-MAC technique uses a finite-difference scheme for the mathematical analysis of the fluid flow problems. Like most numerical techniques, it first divides the system, which is the configuration of the tundish under consideration, into a number of volume elements. Then a set of imaginary markers is introduced into the system to represent the location of the fluid at any instant. The velocity field of the moving fluid domain can be calculated by the application of fluid dynamics principles. Next, the markers are moved according to the calculated velocity field in order to represent the new location of the fluid domain. The procedure can be repeated from the beginning when the tundish is empty until it is filled to a predetermined height in the tundish. The mathematical model was first tested on a one fourth scale water model and good consistency was observed when the simulated filling patterns were compared with the water model experiments. Inclusion distribution and the amount of "dirt" exiting through the outlets of the various strands in the tundish were also analyzed by a fluid particle method. The simulated results showed that for the tundish, inclusion contamination was not uniform at the different strands, which was also confirmed by actual experience in the plant.

Odenthal et al. $^{32)}$ reported results of digital particle image velocimetry (DPIV) on a one fourth scale model of a single strand tundish. It lead to a good interpretation of the dynamic flow phenomena. The flow separated at the bottom of the tundish and a recirculating region was developed. Si- multaneously, the jet out of the shroud induced a contra-rotating double vortex and a short circuit flow around the side walls. A two equation model was developed in order to describe turbulence and the results obtained with this model corresponded well with the DPIV data. They concluded that DPIV can be used as an effective tool to determine two dimensional velocity fields and transient velocity fields. They also suggested the use of 3D DPIV and Laser Doppler Anemometry (LDA) in future. LDA has an advantage that it can measure turbulent fluctuations. A transient two fluid model was developed by Sheng and Jonsson ${ }^{33)}$ to simulate fluid flow and heat transfer in a non isothermal water model of a continuous casting tundish. Thermal stratification in the bath was evident and the results predicted by the two fluid approach adopted, made the effect of natural convection more clear compared with the generally used single fluid $\kappa-\varepsilon$ model. The overvaluation of the conductive heat transfer in the transition region of the system found by using the single fluid approach was eliminated by using the two fluid $\kappa-\varepsilon$ model. The two fluid approach was also found better in describing the counter gradient diffusion phenomenon caused by the thermal buoyancy force.

Ann et $a{ }^{34)}$ predicted a concentration change during grade transition operations during steel thin slab casting based on computer simulations, a water model and plant trials. Fluid flow and mixing patterns in various tundish levels and flow rates were analyzed through $3 \mathrm{D}$ mathematical modelling. They correlated their results with water model experiments and developed a simple, efficient and accurate computational model which could predict the concentration profile at the outlet of the tundish. Based on the model, mixing in/below the mold was analyzed considering EMBR (electro magnetic breaking). The total amount of mixed grade steel only depended on the mixing in the tundish when EMBR is applied. They also concluded that optimum tundish operating condition to minimize intermixed slabs greatly depends on tundish geometry. A systematic study was conducted by Sinha et al. ${ }^{35)}$ of the National Metallurgical Laboratory in India on the fluid flow behavior in a two strand tundish under conditions of submerged entry nozzle (SEN) and open entry nozzle (OEN) with different levels of water in the tundish. Flow was characterized by the profiles of tracer responses at the tundish outlets. Two configurations of entry nozzle (ladle shroud), the straight tube (for SEN and OEN) and the T-shaped (for SEN only) were applied. At $34 \mathrm{~mm}$ immersion depth of the straight ladle shroud, the optimal level of mixed flow in the tundish was produced and thus uniformity in composition was obtained. With the OEN configuration the more was the height of the entry nozzle, the less was the dead volume and the mixing increased.

Sahai $^{36)}$ made a summary on modelling of melt flows in continuous casting tundishes in the Brimacombe Memorial Symposium. He emphasized the importance of proper melt flow in tundishes for the production of high quality clean steel and that this can be achieved by good tundish design and optimum volumetric flow rate of liquid metal. In a full scale model, the Reynolds and Froude similarities can be satisfied simultaneously. However, in a reduced scale model, both similarities cannot be satisfied for a water model. Thus, only Froude similarity is sufficient and con- 
venient similarity criterion for water modelling. In non isothermal systems where buoyancy forces become important, the tundish Richardson number is the sufficient and necessary similarity criteria for modelling. Sahai also mentioned that the solution of the turbulent Navier-Stokes equation with an appropriate set of boundary conditions provides detailed information about the velocity and turbulence fields in the tundish. A coupled solution with the heat transfer equation also provides the temperature field existing in the melt.

Robert and Mazumdar ${ }^{23)}$ developed a steady state, three dimensional, turbulent flow model for analyzing melt flow and RTD in a steelmaking tundish system. They used a control volume based, finite difference procedure and the SIMPLE algorithm. To ensure that their model is consistent and sufficiently robust, they tested the model on several standard systems and obtained satisfactory results. Accordingly, the turbulent model was applied to simulate flow and RTD in four different tundishes. The results were compared with equivalent water model experiments. Except for the single strand tundish system, large differences between measurements and prediction were noted for the other three tundish geometries. Numerical modelling of RTD in the rectangular, six strand tundish system embodying the low Reynold's number $\kappa-\varepsilon$ model of Launder and Jones was found to produce estimates of RTD parameters that are in reasonable agreement with the corresponding experimental results and superior to those deduced via the high Reynold's number $\kappa-\varepsilon$ model.

Ferro et al. ${ }^{37)}$ presented mathematical models for the evaluation of residence time distribution curves for a large variety of vessels. They introduced a new volume called convection diffusion volume to obtain a good representation of RTD curves. Two numerical models for simulation of RTD curves in different vessels were presented. The comparison of measured RTD curves with numerical results from the proposed model shows that these models can successfully represent the general behavior of a fluid inside a variety of systems. The first of the two models proved to be efficient to describe most of the one-peaked RTD curves, in spite of its simplicity. The second one, slightly more complex, represented successfully all the different RTD curves under consideration including those with double peaks. The key feature of these models was the use of a new type of volume, the convection diffusion volume. In order to find the parameters of the model for a given experimental RTD curve, a numerical algorithm was developed and also some simple mathematical relations were found, that allowed the estimation of the parameters of the model from the characteristic parameters of the RTD curve.

Gardin et al. $^{38)}$ carried out an experimental and numerical CFD study of turbulence in a tundish container. Extensive mean and fluctuating velocity measurements were performed using LDA in order to determine the flow field and these data formed the basis for the numerical model validation. CFD modelling of this problem apparently seems easy but actually it was not. Accurate description of the jet is the most important and requires a localized fine grid, but also a turbulence model that predicts the correct spreading rates of the jet and impinging wall boundary layers. The velocities in the bulk of the tundish are generally much smaller than those of the jet, leading to damping of turbulence, or even laminar flow. They started the work with an objective of applying and validating the $\kappa-\varepsilon$ model of Wilcox to a tundish flow problem. This model was thought to have advantages over the $\kappa-\varepsilon$ model. After initial 2D studies they found that the $\kappa-\varepsilon$ model was diffusing the wall jet too quickly. Medium grid density 3D computations, confirmed this finding. The authors developed several low-Reynolds number $\kappa-\varepsilon$ model variants to compute the flow and compare against measurements. The $\kappa-\varepsilon$ modifications proved to be very successful, with the newly developed $\kappa-\varepsilon$ D1 model being the best. This indicated there is no need to use more sophisticated differential Reynolds stress models, which are more difficult and take longer to converge. From the grid refinement they concluded that certain turbulence models were more grid-sensitive than others. They also concluded that the LES model might still have certain limitations for practical use.

Guthrie and $\mathrm{Isac}^{2)}$ reported on the importance of the use of full-scale models for studying the fluid flow and transport phenomena in ladle-tundish-mold operations. They mentioned that reduced scale models are convenient to simulate first order simulations of inertially dominated flows, but the finer details are better analyzed in full scale models where Re, Fr and Tu can be simultaneously respected.

Jha and Dash ${ }^{39)}$ employed different turbulence models to the design of optimum steel flows in a tundish. The Navier-Stokes equation and the species continuity equation were solved numerically in a boundary fitted coordinate system comprising the geometry of a large-scale industrial size tundish. The solution of the species continuity equation predicted the time evolution of the concentration of a tracer at the outlets of a six-strand billet caster tundish. The numerical prediction of the tracer concentration using six different turbulence models, [the standard $\kappa-\varepsilon$, the $\kappa-\varepsilon$ RNG, the low Re number Lam-Bremhorst model, the Chen-Kim high Re number model (CK), the Chen-Kim low Re number model (CKL) and the simplest constant effective viscosity model (CEV)] which compared favourably with that of the experimental observation for a single strand bare tundish. It was found that the overall comparison of the $\kappa-\varepsilon$ model, the RNG, the Lam-Bremhorst and the CK model was much better than the CKL model and the CEV model as far as gross quantities like the mean residence time and the ratio of mixed to dead volume were concerned. However, the $\kappa-\varepsilon$ model predicted the closest value to the experimental observation compared to all other models. The prediction of the transient behaviour of the tracer was best done by the Lam-Bremhorst model and then by the RNG model, but these models did not predict gross quantities very accurately as did the $\kappa-\varepsilon$ model for a single strand bare tundish. With the help of the above six turbulence models, mixing parameters, such as the ratio of mix to dead volume and the mean residence time, were computed for the six strand tundish for different outlet positions, height of advanced pouring box (APB) and shroud immersion depth. It was found that three turbulence models show a peak value in the ratio of mixed to dead volume when the outlets were placed at $200 \mathrm{~mm}$ away from the side wall as shown in Fig. 1 below.

An advanced pour box (APB) was put on the bottom of 


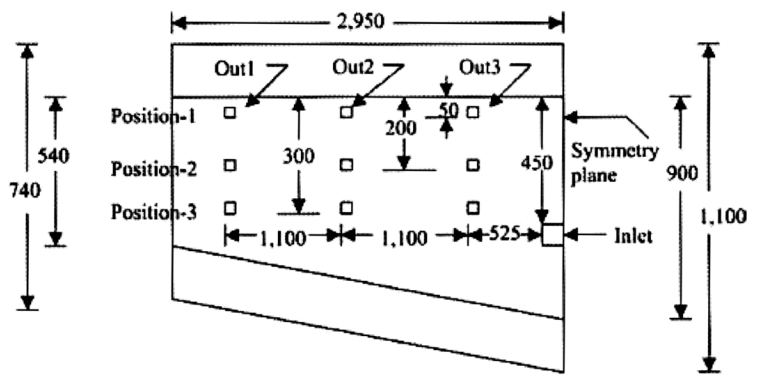

Fig. 1. Top (plan) view of the tundish showing outlets on the bottom plane.

the tundish surrounding the inlet jet when the outlets were kept at $200 \mathrm{~mm}$ away from the wall. It was also found that there exists an optimum height of the APB where the ratio of mixed to dead volume and the mean residence time attain further peak values signifying better mixing in the tundish. At this optimum height of the APB, the shroud immersion depth was made to change from 0 to $400 \mathrm{~mm}$. It was also observed that there exists an optimum immersion depth of the shroud where the ratio of mix to dead volume still attains another peak signifying slightly better mixing. However, none of the turbulence models predicts the same optimum height of the APB and the same shroud immersion depth, as the optimum depth. The optimum height of the APB and the shroud immersion depth were decided when two or more turbulence models predicted the same values. Jha et al. ${ }^{16)}$ also performed large eddy simulation to study mixing in a tundish. They took the solution of the $\kappa-\varepsilon$ model as a starting guess for the large eddy simulation (LES). A solution for the LES could be arrived at after adapting a local refinement of the cells (twice), so that the near wall $y^{+}$could be set less than 1 . Such a refined grid gave a time independent solution for the LES, which was used to solve the species continuity equation. The LES solution slightly over predicted the mean residence time but could predict fairly well the mixed volume. However the LES was unable to predict both the peaks in the tracer concentration like the $\kappa-\varepsilon$, RNG and Lam-Bremhorst models were able.

Lopez-Ramirez et al. ${ }^{10)}$ studied the influence of input temperature changes on molten steel flow in tundishes by physical and mathematical modelling. In their study, the difference between considering and not considering the thermal influence changed the parameter values in approximately $5 \%$ of the relative standard deviation. The thermal response obtained from an input step temperature change reproduced the real behavior of the molten steel flow in a tundish dominated by buoyancy forces. A comprehensive process model for fluid flow and heat transfer in the tundish was developed by Pardeshi et al. ${ }^{40)}$ which was capable of capturing transient process dynamics. The model was based on a conjugate thermal analysis of the tundish, accounting for simultaneous heat transfer through the liquid steel, refractory, steel shell and dams. The model was validated by comparing model predictions for laminar and turbulent fluid flow conditions cited in the literature. Plant campaigns were undertaken to collect plant data for tuning and validation of the model. Kumar et al. $^{26)}$ from their experimental and computational study on flow modelling and RTD, indicated that a sufficiently small grid resolution (control vol-
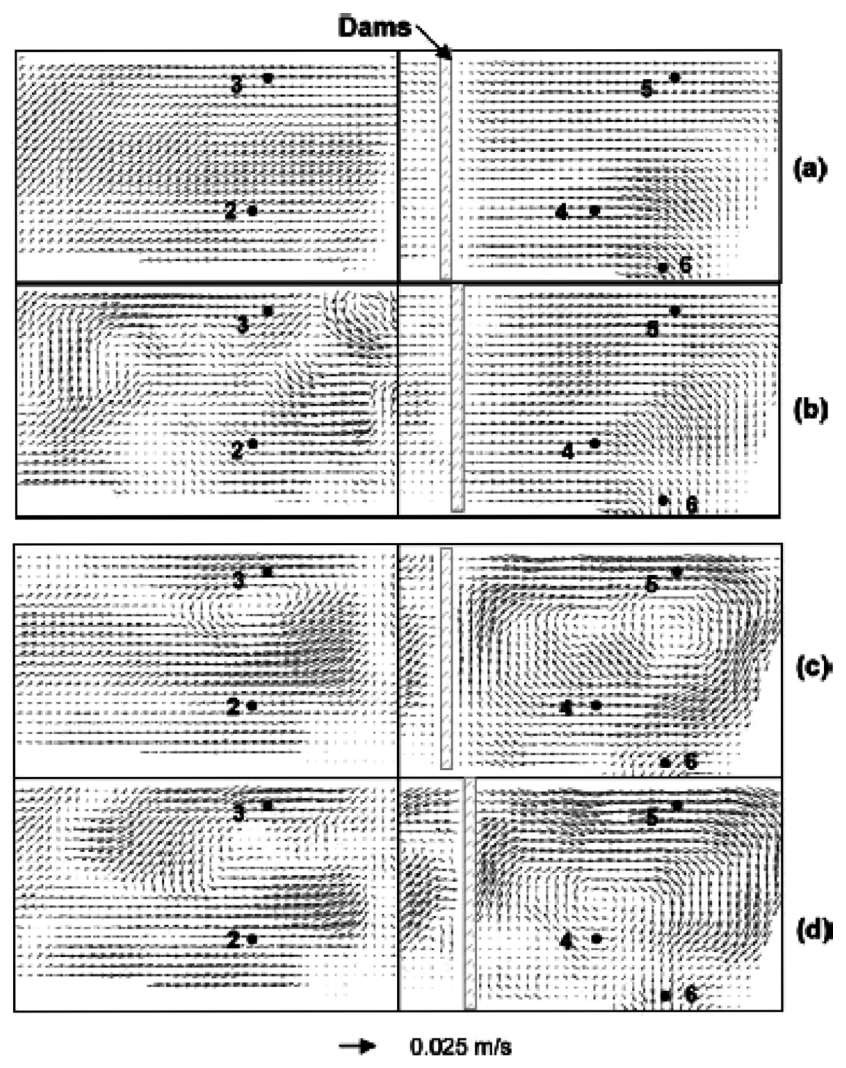

Fig. 2. Velocity fields of water under isothermal conditions; the right side is before the gas curtain and the left side is after the gas curtain: (a) without gas, (b) gas flow rate $246 \mathrm{cc} / \mathrm{min}$, (c) gas flow rate $580 \mathrm{cc} / \mathrm{min}$, and (d) gas flow rate $912 \mathrm{cc} / \mathrm{min}$.

ume of the order of $10^{-6} \mathrm{~m}^{3}$ ) is necessary to arrive at a practical grid independent solution. They also reported that the Reynolds Stress model was found to simulate RTD in the system somewhat better to the standard coefficient $\kappa-\varepsilon$ model. Their mathematical model was validated with experimental results and proved sufficiently robust and reliable to predict mixing parameters in tundishes with and without flow control devices.

Zamora et $a l .{ }^{41)}$ studied the inertial and buoyancy driven water flows under gas bubbling and thermal stratification conditions in a tundish model. Steel flow dominated by inertial and buoyancy flows under gas bubbling and thermal stratification conditions, in a one-strand tundish, was studied, using a $2 / 5$ scale water model. The use of a turbulence inhibitor yielded plug flow volume fractions well above $40 \%$ for a casting rate of 3.12 tons $/ \mathrm{min}$ under isothermal conditions. Small flow rates of gas injection $\left(246 \mathrm{~cm}^{3} / \mathrm{min}\right)$, through a gas curtain, improved the fluid flow by enhancing the plug flow volume fraction. Higher flow rates originated an increase in back-mixing flow, forming recirculating flows on either side of this curtain. Step inputs of hot water drove streams of this fluid towards the bath surface due to buoyancy forces. A rise in gas flow rate led to a thermal homogenization in two separated cells of flow located at each side of the gas curtain. Step inputs of cold water drove streams of fluid along the tundish bottom. Use of the gas curtain homogenized the lower part of the tundish as well as the upper part of the bath at the left side of the curtain. Figure 2 shows the above phenomena clearly. However, 
temperature at the top corner of the tundish, in the outlet box, remained very different than the rest of the temperatures inside this tundish. High gas flow rates $\left(912 \mathrm{~cm}^{3} / \mathrm{min}\right)$ were required to homogenize the bath after times as long as twice the mean residence time of the fluid. Particle image velocimetry (PIV) measurements corroborated the formation of recirculating flows at both sides of the gas curtain.

Ramos-Banderas et al. ${ }^{42)}$ performed mathematical simulations and physical modelling of unsteady fluid flows in a water model tundish mould. The LES approach used by them was able to predict qualitatively the instantaneous upper recirculating flow fields of water in the physical model. Agreement of simulated and measured jet parameters such as the jet angle and the impingement position of the entry jets in the narrow wall of the mould were acceptably good. Thus they suggested that the LES approach is well recommended to estimate jet characteristics. Changes of flow pattern with time were generated as a result of the vertical oscillation motion of the jet core. This motion was promoted by the residual Reynolds stresses that characterize turbulent flow. The fluid flow pattern in the jet root was unaltered by changes of the flow rate of the liquid. The asymmetry of fluid flows caused by these stresses yielded biased flows. Mass transfer in the mould also yielded asymmetric flow patterns as a consequence of the fluid flow characteristics.

Tripathi and Ajmani ${ }^{43)}$ reported on a numerical investigation of fluid flow phenomena in a curved shape tundish (Fig. 3) by numerical investigation. A 3D mathematical model was developed by them. The results were compared with a conventional delta shaped tundish. The strong role of curvature in improving fluid flow characteristics and enhancing inclusion floatation was evident from the results. They also reported a considerable increase in the plug volume and mean residence time with the use of contour shaped pouring chambers as compared to those with sharp corners. The mathematical model was validated with experimental results for a single strand bare tundish.

A novel tundish mixing model was proposed to predict the outlet concentration of the tundish during a grade transition by Cho and Kim. ${ }^{44)}$ To enhance the efficiency and replication performance, the present model was designed to minimize the number of parameters to only one that needs to be tuned for easier application to new situations whereas the Huang and Thomas ${ }^{45}$ ) model had six parameters to be tuned. Two types of water model were employed to verify Cho and Kim's model, and the real grade mixed blooms were produced through a grade transition continuous casting. When the present tundish mixing model was applied to the cases of the water models and real bloom casting, the numerical results of the present model were found to be in good agreement with the experimental data, and the constant parameter $f$ of the present model was found to be determined according to the tundish shape.

Alkishriwi et al. $^{46)}$ carried out large eddy simulations of a continuous casting tundish to investigate the turbulent flow structure and vortex dynamics. The method used an implicit time accurate dual time stepping scheme in conjunction with low Mach number preconditioning and multigrid acceleration. To validate the scheme, large-eddy simulations of turbulent pipe flow at $\mathrm{Re}_{\mathrm{T}}=1280$ and cylinder

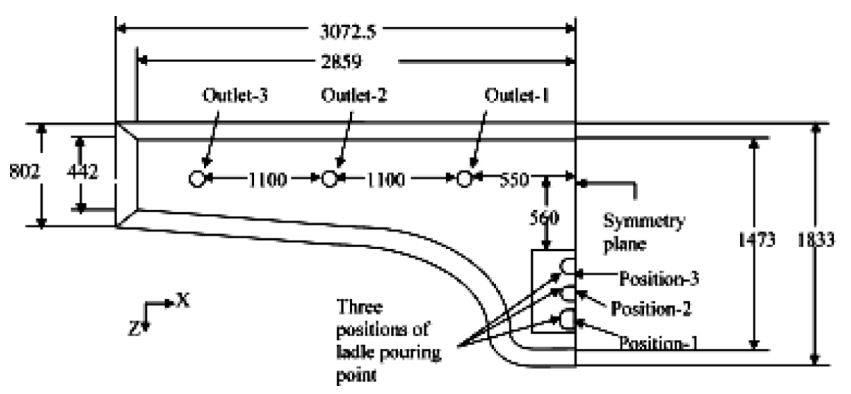

Fig. 3. Top (plan) view of the curved shaped tundish.

flow at $\mathrm{Re}_{\mathrm{D}}=3900$ were performed. The results showed the scheme to be efficient and to improve the accuracy at low Mach number flows. The findings from the LES showed the presence of many intricate flow details that have not been observed before by customary RANS approaches. Fluid flow dynamics during ladle drainage operations of steel under isothermal and non-isothermal conditions were studied using the turbulence shear stress transport $\kappa-\varepsilon$ model (SST $\kappa-\omega)$ and the multiphase volume of fluid (VOF) model by Davila et al. ${ }^{47)}$ At high bath levels, the angular velocity of the melt, close to the ladle nozzle, was small rotating anticlockwise and intense vertical-recirculating flows were developed in most of the liquid volume due to descending steel streams along the ladle vertical wall. These streams ascended further downstream driven by buoyancy forces. At low bath levels, the melt, which was close to the nozzle, rotated clockwise with higher velocities whose magnitudes were higher for shorter ladle stand still times. Figures 4 to 7 show draining operations under isothermal and non-isothermal conditions.

These velocities were responsible for the formation and development of a vortex on the bath free surface, which entrained slag into the nozzle by shear-stress mechanisms at the metal-slag interface. The critical bath level or bath height for this phenomenon was $0.35 \mathrm{~m}$ (in this particular ladle design) for a ladle standstill time of $15 \mathrm{~min}$ and decreased with longer ladle stand still times. At these steps, the vertical-recirculating flows were substituted by complex horizontal-rotating flows in most of the liquid volume. Under isothermal conditions, the critical bath level for vortex formation on the melt free surface was $0.20 \mathrm{~m}$, which agrees very well with that determined with a $1 / 3$ scale water model of $0.073 \mathrm{~m}$. It was concluded that buoyancy forces, originated by thermal gradients, as the ladle cools, were responsible for increasing the critical bath level for vortex formation. Sankanarayan and Guthrie ${ }^{48,49}$ mentioned that the diameter ratio of the outlet nozzle and the ladle is important and this ratio and the critical height for vortex formation are proportional. For a constant ratio of outlet diameter to ladle diameter, the critical height becomes larger with higher initial bath heights. Understanding vortex mechanisms will be useful to design simple and efficient devices to break down the vortex flow during steel draining even at very low metal residues in the ladle. Vayrynen et $a l{ }^{50)}$ modeled steady state and transient casting situations. Their work was focused on tuning and validation of a commercial CFD package which will be used to simulate tundish operations. They concluded that the software was not able to simulate the downscaled water model accurately, 


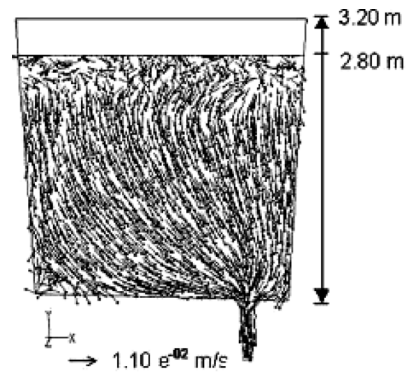

(a)

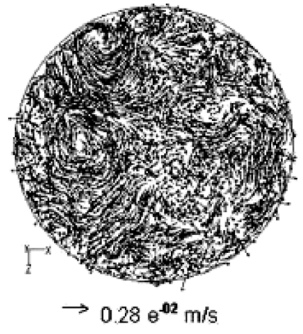

(b)
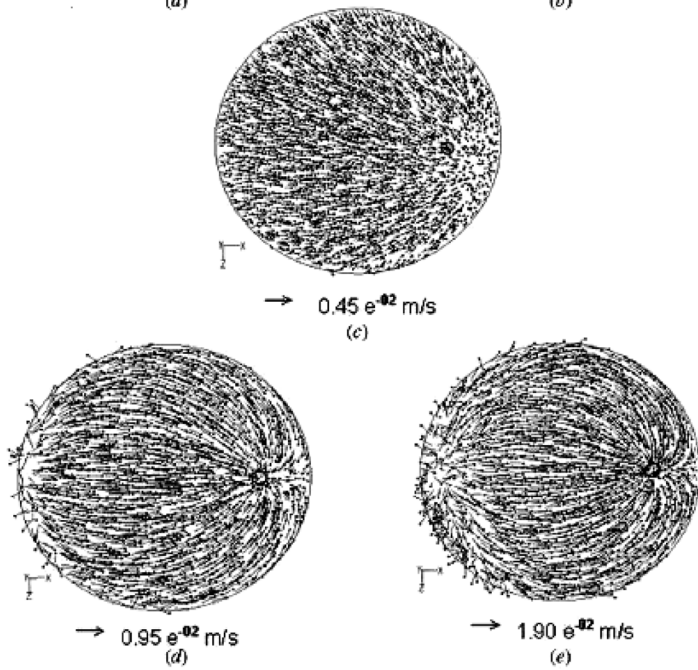

Fig. 4. Velocity fields in different planes during the isothermal drainage of liquid steel from a ladle to a tundish at a throughput of $2.1 \mathrm{ton} / \mathrm{min}$ for a steel level in the ladle of $2.80 \mathrm{~m}$ : (a) vertical plane, (b) horizontal plane at $2.50 \mathrm{~m}$ from the bottom, (c) horizontal plane at $1.50 \mathrm{~m}$ from the bottom, (d) horizontal plane at $0.35 \mathrm{~m}$ from the bottom, and (e) horizontal plane at $0.20 \mathrm{~m}$ from the bottom.

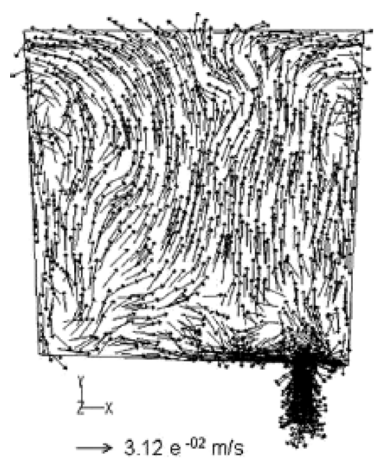

(a)

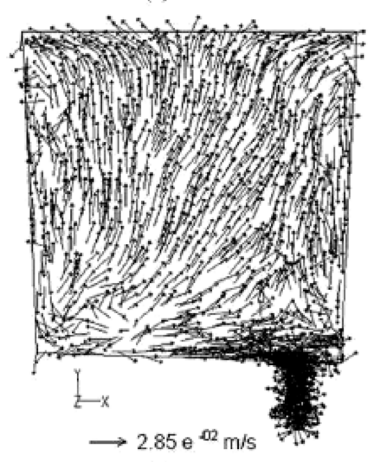

(c)

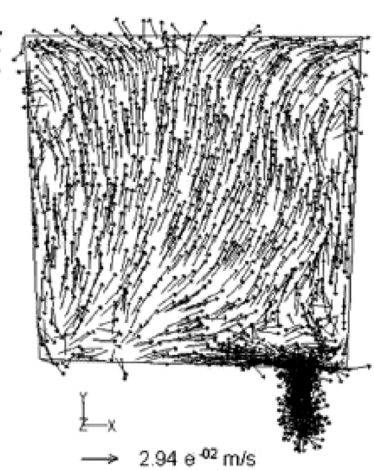

(b)

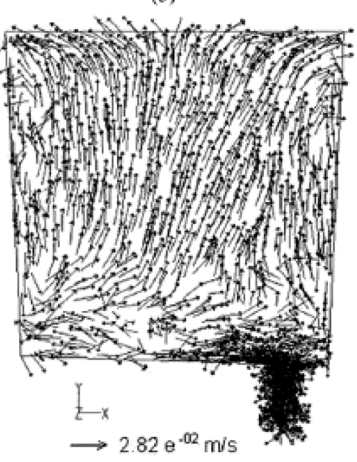

(d)
Fig. 5. Velocity fields developed during thermal stratification of liquid steel in a ladle for different standstill times of the ladle: (a) $15 \mathrm{~min}$, (b) $30 \mathrm{~min}$, (c) $45 \mathrm{~min}$, and (d) $60 \mathrm{~min}$.
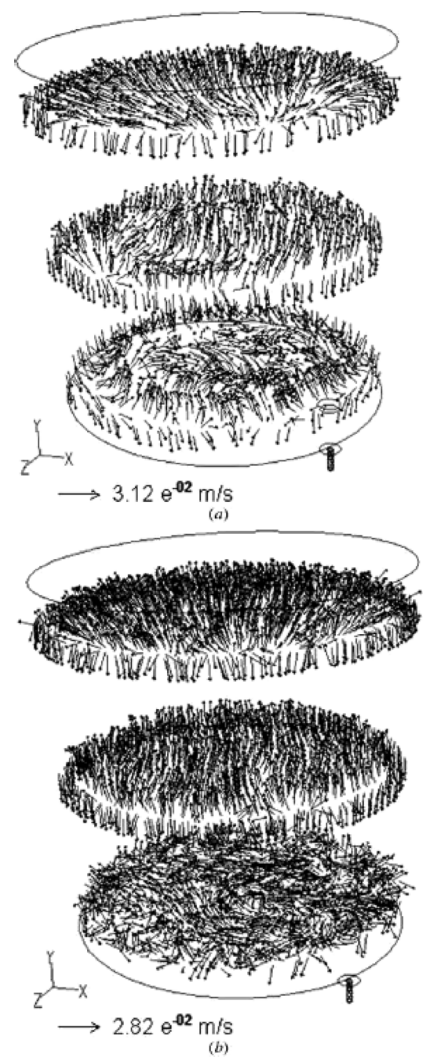

Fig. 6. Views of velocity fields at different horizontal planes located at 2.80, 1.50, and $0.30 \mathrm{~m}$ from the bottom of the ladle: (a) after a standstill time of $15 \mathrm{~min}$ and (b) after a standstill time of $60 \mathrm{~min}$.
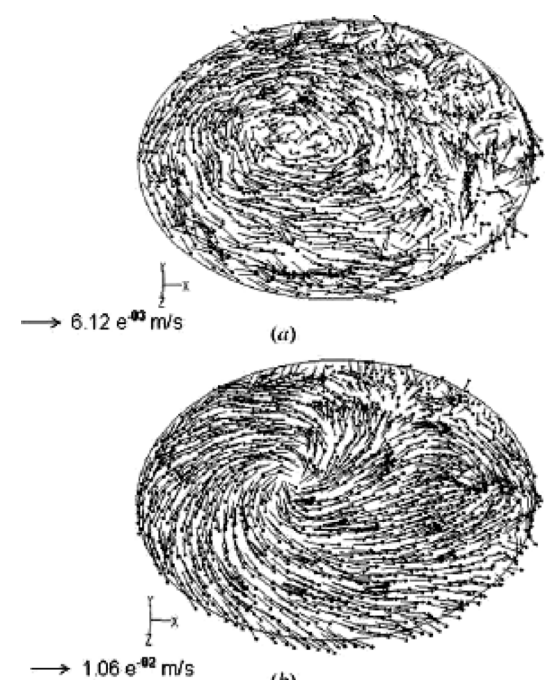

(b)

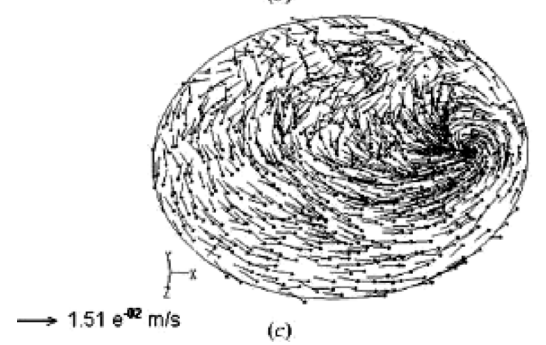

Fig. 7. Velocity fields at horizontal planes during non-isothermal drainage of liquid steel from a ladle at different horizontal planes for a bath level of $1.50 \mathrm{~m}$ : (a) $0.80 \mathrm{~m}$ from the bottom, (b) $0.40 \mathrm{~m}$ from the bottom, and (c) $0.20 \mathrm{~m}$ from the bottom. 
but on the other hand both temperature and transient casting situation results of the full scaled tundish were satisfactory. They mentioned that one possible reason for this behavior is that areas of clearly turbulent flow in the water model are much smaller than in theactual tundish and this transition of flow type is very difficult for the present turbulence models to calculate accurately. Zorzut et al. ${ }^{51)}$ developed a mathematical model which could predict steel grade change. The model was set up on some dilution interconnecting elementary cells that describe the shroud and each strand. The cells were considered with uniform concentration. Such a model was very useful in the definition of the technological practice for the steel grade change operation.

Inclusion removal in the tundish is a very important operation and a lot of work has been in literature till date. Mazumdar and Guthrie ${ }^{1)}$ had discussed the studies on inclusion separation where Stokes terminal rising velocity of the inclusion particles were vectorially added to the vertical component of the fluid motion in the partial differential equation which is basically a convection-diffusion equation for species transfer.

$$
\begin{aligned}
& \frac{\partial\left(\rho c_{i}\right)}{\partial t}+\frac{\partial\left(\rho u c_{i}\right)}{\partial x}+\frac{\partial\left(\rho\left[v+u_{T, i}\right] c_{i}\right)}{\partial y}+\frac{\partial\left(\rho w c_{i}\right)}{\partial z} \\
& =\frac{\partial}{\partial x}\left(\Gamma_{\text {eff }} \frac{\partial c_{i}}{\partial x}\right)+\frac{\partial}{\partial y}\left(\Gamma_{\text {eff }} \frac{\partial c_{i}}{\partial y}\right)+\frac{\partial}{\partial z}\left(\Gamma_{\text {eff }} \frac{\partial c_{i}}{\partial z}\right)+S_{i}
\end{aligned}
$$

After obtaining a steady state velocity field Eq. (4.1) is solved for inclusion concentration. Generally each group of inclusions is characterized by their diameter. They also discussed the source term $S_{i}$ that represents generation or destruction of a particular size of inclusion by coalescence and the boundary conditions. Details of these are not repeated here. Recent studies showed the use of different models to study inclusion separation phenomenon. Hamill and Lucas $^{52)}$ used an algebraic slip model (ASM) which basically is a simplified form of a multi-phase model to study inclusion motion and its removal. Assuming that the small particles always move at their slip velocity, which basically comes from a balance between the buoyancy and drag force, they solved separate scalar volume fraction equation for each particle species along with the basic conservation equations for multiphase (the equations are not given here). They used software CFX to compute the results and compared their results with previously published results of Joo et al. ${ }^{53)}$ who used Eq. (4.1) which is based on single phase modelling approach. Joo et al. ${ }^{53)}$ used the METFLO 3D software (a code developed in McGill university) to solve those equations. For the flow conditions modelled, they showed that most inclusions greater than 100 microns should float out, independent of flow control devices while all those less than 40 microns would not be helped by flow modifying devices, and be retained in liquid steel exiting to the strands. More recently Huelstrung et al. ${ }^{55)}$ studied the influence of tundish volume and Schwarze et al. ${ }^{54)}$ studied the influence of different weir \& dam arrangement on predicting the degree of inclusion separation. Huelstrung et $a l .{ }^{55)}$ used "discrete phase model" to predict the degree of inclusion separation and used the results as a tool for designing a higher capacity tundish. Better macroscopic cleanliness of steel slabs produced during abnormal casting condition caused due to stopping of one of the strand slab caster led them to design a tundish with a higher volume. They investigated the behaviour of solid alumina particles using a Euler-Lagrangian method. In this method the particle trajectories were computed in a Lagrangian reference frame. They solved the basic conservation equations along with the following equation.

$$
\frac{d u_{p}}{d t}=F_{D}\left(u-u_{p}\right)+\frac{g_{x}\left(\rho_{p}-\rho\right)}{\rho_{p}}+F_{x}
$$

The coupling between continuous phase steel and discrete phase alumina was done by defining the drag force $\left(F_{D}\right)$ according to the following equation assuming the particle to be in the Stokes regime.

$$
F_{D}=\frac{18 \mu c_{D} \operatorname{Re}}{24 \rho_{p} d_{p}^{2}}
$$

Where

$$
\operatorname{Re}=\frac{\rho d_{p}\left|u_{p}-u\right|}{\mu}
$$

and

$$
C_{D}=a_{1}+\frac{a_{2}}{\operatorname{Re}}+\frac{a_{3}}{\operatorname{Re}^{2}}=\frac{24}{\operatorname{Re}}
$$

$F_{x}$ is an additional acceleration term. It is the "virtual mass" force, i.e. the force required to accelerate the liquid surrounding the particle.

Schwarze et al. ${ }^{54)}$ in their isothermal and non isothermal studies of a V-shaped tundish, also considered the effect of added mass (Fig. 8), which should be included in case of an accelerating or decelerating particle. Apart from that, they also included a term for the force of excess pressure differential with acceleration of the fluid motion.

$$
\frac{d u_{p}}{d t}=F_{D}\left(u-u_{p}\right)+\frac{g_{x}\left(\rho_{p}-\rho\right)}{\rho_{p}}+F_{x}+F_{p} \ldots
$$

For uniform flow field it was found,

$$
F_{p}=\frac{\rho}{\rho_{p}}\left(u_{p} \frac{d u}{d x}\right)
$$

To simulate the chaotic effect of the turbulence eddies of the liquid phase on the inclusion trajectories; a discrete random-walk model was applied by Diaz et $a .^{28)}$ In this

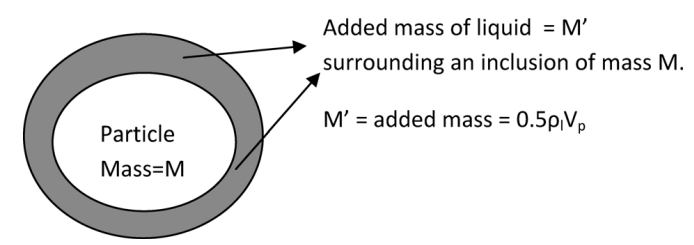

Fig. 8. Illustrating the "added mass" associated with a film of liquid surrounding the particle, or inclusion. 
model, a fluctuant random-velocity vector $\left(u_{i}^{\prime}\right)$ is added to the calculated time-averaged vector $\left(u_{i}\right)$, in order to obtain the inclusion velocity $\left(u_{i}\right)$ at each time step, as particles travel through the fluid. Each random component of the inclusion velocity is proportional to the local turbulent kinetic energy level $\left(\kappa_{\mathrm{p}}\right)$, according to the following equation:

$$
u_{i}^{\prime}=\zeta_{i} \sqrt{\bar{u}_{i}^{\prime 2}}=\zeta_{i} \sqrt{\frac{2 \kappa}{3}}
$$

where $\zeta_{i}$ is a random number, normally distributed between -1 and 1 , which changes at each integration step.

In considering the model equation for particle tracking Diaz et $a{ }^{28)}$ considered the following equation instead of [Eq. (4.2)].

$$
\frac{d v_{p, j}}{d t}=\frac{18 \rho v}{\rho_{p} d_{p}^{2}}\left(1+0.15 \operatorname{Re}_{p}^{0.687}\right)\left(u_{i}-v_{p, i}\right)+\left(1-\frac{\rho}{\rho_{p}}\right) g_{i} \mid
$$

Zhang et al. ${ }^{56)}$ proposed three modes of inclusion removal from molten steel in the tundish viz. floatation to the free surface, collision and coalescence of inclusions to form larger ones and adhesion to the lining solid surfaces. They studied the 3D fluid flow with and without flow control devices. The results indicated that flow control devices effectively control the strong stirring energy within the inlet zone. Flow control devices were also favorable for inclusion removal. The total removal ratio was $51 \%$ without flow control devices where inclusions with radii greater than 72 microns were totally removed. This increased to $79 \%$ with flow control devices where inclusions with radii greater than 61 microns were totally removed. Out of this $79 \%$, removal by floatation was $49.5 \%$ and removal by adhesion was $29.5 \%$. The collision and coalescence mode was a better way to remove smaller size inclusions as the number of collisions per unit time per unit volume of steel was much higher for smaller inclusions than bigger inclusions.

Javurek et al. ${ }^{57)}$ considered the removal of non metallic inclusions due to buoyancy forces in continuous casting tundishes. They found the reasons why the particle separation is worse than the calculated maximal possible removal rate. The reasons were unsuitable fluid flow pattern and turbulent particle diffusion. They also concluded that RTD curves are inappropriate to estimate the particle separation in tundishes. Use of direct calculation and CFD simulation was recommended.

Schwarze et al ${ }^{54)}$ studied the degree of inclusion separation in different ( $\mathrm{V}$ shaped) tundish configurations by numerical modelling. The numerical model was based on the Euler-Lagrange approach. The flow was described by the Reynolds averaged transport equations for mass, momentum and energy in conjunction with the RNG $\kappa-\varepsilon$ model. They considered both isothermal and non isothermal flows. Only small differences between the isothermal and non isothermal flows were observed for the conditions modelled. Thermal natural convection was not that significant. The results of the numerical model were in good agreement with corresponding data from water model studies. The highest degree of inclusion separation was reached when a

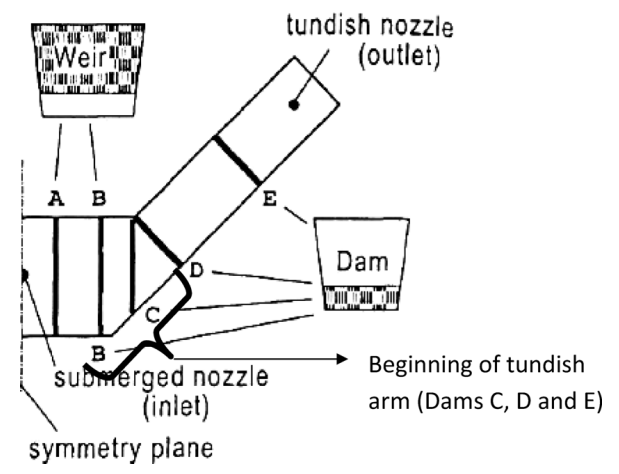

Fig. 9. Illustration of a V-shaped tundish with different arrangements of a weir and dams.

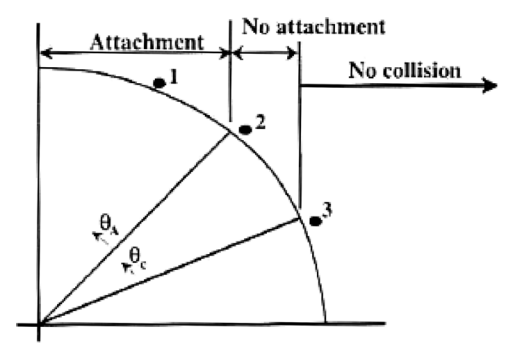

Fig. 10. Schematic diagram of critical impingement angles.

dam was positioned just at the beginning of the tundish arm (Fig. 9).

Thomas and $\mathrm{Bai}^{58)}$ summarized the formation mechanisms, detection methods and prevention of tundish nozzle clogging, focusing on the role of computational models in quantifying the non-composition-related aspects. They classified tundish clogging into four main types viz. the transport of oxides present in the steel to the nozzle wall, air aspiration into the nozzle, chemical reaction between the nozzle refractory and the steel and steel solidified in the nozzle. However in practice a clog can be a combination of two or more of the above types. They mentioned that clogging can be best detected during casting by simultaneous monitoring of several different parameters like argon back pressure, nitrogen pickup, mold level fluctuations and flow control position relative to casting speed. Solutions to clogging problems were also mentioned by them. They are minimizing inclusions by improved steelmaking practices, optimizing fluid flow and transfer processes, controlling steel alloy additions, slag and refractory compositions, improving nozzle material design and avoiding air aspiration. Rogler et al. ${ }^{59}$ reported the probability of inclusion removal in a tundish by gas bubbling. They developed a simple mathematical model and concluded that the probability of particle/bubble attachment $\left(P_{a t}\right)$ is the product of the probabilities of three fundamental steps of this process including thinning, rupture and three-phase contact stability ${ }^{60)}$ (Fig. 10).

However, only the probability of film thinning can be calculated by any known method and they suggested that the probability of the last two steps could be assumed to be unity. $P_{a t}$ increases with decreasing particle size and increases with increasing bubble size. But for attachment collision is necessary and hence the particle collection probability $(P)$ is the product of $P_{a t}$ and the collision probability $P_{c}$. A semi-analytical solution of the Navier-Stokes equation gives a rough estimate of $P_{c}$. This analysis shows that 
the collection probability $(P)$ increases with decreasing bubble size and increases with increasing particle size. The removal of exogenous non-metallic inclusions by optimization of hydrodynamic characteristics was reported by Kuklev et al. ${ }^{61)}$ The suggested methods are increasing tundish size to allow more residence time and the use of flow control devices. Rogler et $a .^{62)}$ performed physical modelling of inclusion removal in a tundish by gas bubbling. They used water as the analogue of steel and linear low density polyethylene (LLDPE, $\rho=0.92$ ) as an analogue of inclusions. They concluded that separation efficiency of inclusion particles within the flowing liquid bath in a tundish is influenced by a number of factors like overall fluid flow behavior, chemical and physical nature of the inclusion, size of the inclusions, and rates and mechanisms of particle capture by various potential particle sinks. From their study they showed that FCD enhanced particle separation efficiency and properly sized bubbles induced the highest particle separation efficiencies.

An extensive review of the literature shows a large number of modelling efforts covering various aspects, such as fluid flow, RTD, effects of FCD on flow pattern, inclusion removal and thermal energy transport etc., have been reported. However, most of the modelling did not consider refractories, slag, and fluxes; in their predictions. Solhed et $a{ }^{24)}$ included these in their modelling effort, and studied slag-steel interactions in continuous-casting tundishes. A model was developed that took into account the steel, slag and refractory phases. The model was also used to determine the optimal location of flow devices, making the temperature distribution in the steel more even and enhancing the removal of inclusions to the slag. In that study, the focus was to study the slag/steel interface. Predictions showed that slag is dispersed into the steel close to the interface, as well as close to the ladle shroud. A momentary interfacial solidification sampling (MISS) method was developed to confirm these predictions. Analysis of the samples by ultrasonic testing, optical microscopy and SEM confirmed the presence of non metallic particles close to the slag steel interface and close to the ladle shroud. The analyses also showed that the slag steel interface is very irregular despite low velocities. They concluded that the slag/steel interface is unstable and the liquid steel at the interface can engulf slag and it may lead to the formation of inclusions in the final cast product. An investigation of slag floatation and entrapment was studied by Solhed et $a l^{25)}$ using fluid flow simulations, sampling and physical metallurgy. A model of a continuous casting tundish, which besides the steel phase also considers refractory, slag and flux was developed and presented together with the basic ideas behind the development. The model was verified with velocity and temperature measurements in steel and refractory. The agreement between the measured velocities and temperatures and the corresponding predictions were good. Solhed et al. ${ }^{25)}$ solved the species conservation equation for each phase apart from the conservation of mass, conservation of momentum and conservation of energy equation.

$$
\rho \cdot \vec{V} \cdot \frac{D n_{\alpha}}{D t}=\nabla \cdot\left(\sum_{j} \rho D_{j} \cdot n_{\alpha, j} \cdot \nabla m_{j}\right) \mid \ldots
$$

They incorporated the slag phase in their model.

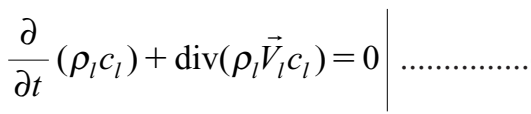

$$
\begin{aligned}
& \frac{\partial}{\partial t}\left(\rho_{s} c_{s}\right)+\operatorname{div}\left(\rho_{s} \vec{V}_{s} c_{s}\right)=0
\end{aligned}
$$

The relative volumes of steel and slag in a particular control volume are given by the following equations.

$$
\begin{gathered}
V_{\text {steel }}=\frac{c_{l}}{c_{l}+c_{s}} \frac{1}{\rho_{\text {steel }}} . \\
V_{\text {slag }}=\frac{c_{l}}{c_{l}+c_{s}} \frac{1}{\rho_{\text {slag }}} .
\end{gathered}
$$

From their computational results it was seen that slag concentrations were very low in the regions near the walls, where flows were upwardly directed, but that near to the shroud and the stopper rod, slag concentrations were higher and the downwardly directed velocity components caused the slag to penetrate the steel. It is evident that not much work has been done on slag entrainment. So more work on slag entrainment using both physical and mathematical modelling needs to be done. Here at the MMPC modelling slag entrainment both physically and numerically is being done by some researchers. In the numerical model, the VOF approach is currently used.

The effect of flow control devices have also been extensively studied by a number of researchers. ${ }^{18,63-68, e t c .)}$ The research on FCD has been so vast in the past decade that perhaps it needs a separate review on the effect of FCD on performance of tundish. However it has been seen that FCD have a positive effect on tundish performance if their design is properly optimised and positioning in the tundish is proper. But for inclusion removal FCD have failed to show their efficiency either in physical or mathematical modelling. ${ }^{69-71)}$ Also improper positioning of FCD may result in detrimental flow patterns inside the tundish. Also in large tundishes which allow sufficient residence time for inclusion floatation, FCD are not recommended because they are inconvenient to sustain a hot cycle tundish practice, which is quite economical. With a large tundish, the deep melt bath does not require a pour pad either. ${ }^{8)}$

A "swirling ladle shroud" (SLS) may be very useful to reduce turbulent kinetic energy within the inlet zone. A new design of ladle shroud, obtained through water modelling, that controls turbulence of the entry jet in continuous casting tundishes has been proposed by Diaz et al. ${ }^{72)}$ Particle Image Velocimetry (PIV) measurements indicated that this design decreased the impact velocity on the tundish bottom close to $1 / 3$ of that provided by a conventional ladle shroud. This achievement was due to a swirling jet that promoted a recirculatory flow in the horizontal planes of the tundish. The swirling effects helped to dissipate the turbulence energy of the jet before it hit the tundish bottom, thereby decreasing fluid velocities impacting the back and front walls of the tundish.

Turbulence models such as $\kappa-\varepsilon, \kappa-\omega$ and RSM were applied to simulate the experimental PIV measurements of 


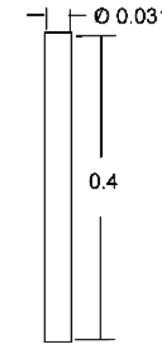

a)

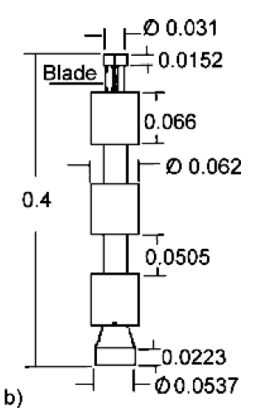

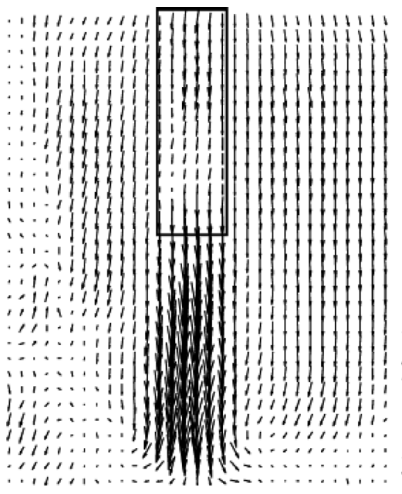

(a)

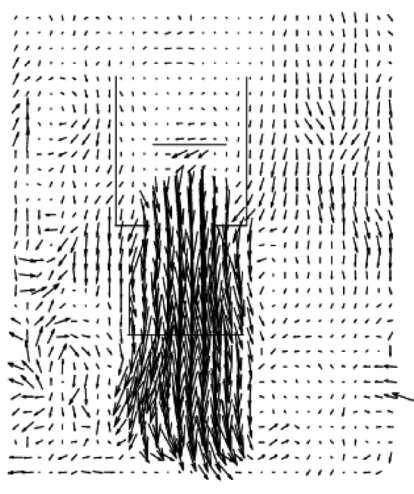

(b)

Fig. 12. Swirling motion of the entry jet measured by PIV; (a) conventional ladle shroud and (b) SLS.

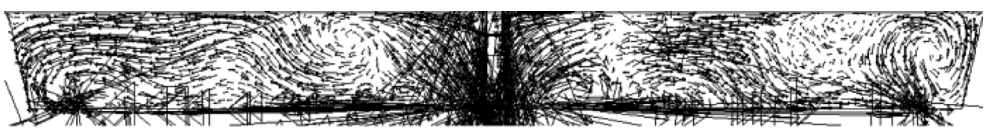

a)

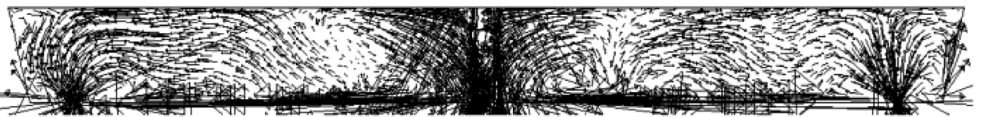

b)

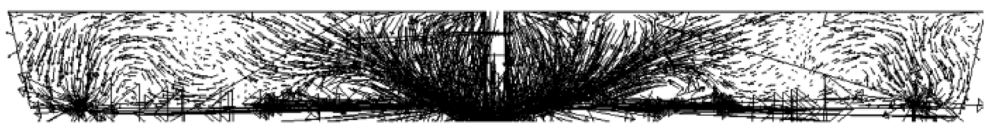

$\rightarrow 0.016 \mathrm{~m} / \mathrm{s}$

Fig. 13. Velocity fields along the symmetrical-longitudinal plane, predicted mathematically, using different models of the turbulence; (a) $\kappa-\varepsilon$ model, (b) $\kappa-\omega$ model, and (c) RSM model.

velocities in the fluid flow. Only the RSM model yielded predictions that agreed remarkably well with the experimental determinations (Figs. 13, 14 and 15). These results were sufficiently good as to avoid the employment of flow control devices such as dams, weirs, turbulence inhibitors and the like, in tundishes.

Hou and $\mathrm{Zou}^{73)}$ studied the flow patterns in a new type swirling flow tundish with a swirling chamber. Numerical simulations suggested that the RNG $\kappa-\varepsilon$ model was more suitable for this new type tundish and can get convergent results more easily than the standard $\kappa-\varepsilon$ model for strong curvature flows and flows with different Reynolds numbers in different areas (Fig. 16). The grid independent feature of this numerical model (RNG $\kappa-\varepsilon$ model) was verified by two kinds of grids with different node densities (Figs. 17(a) and 17(b)). They also concluded that the asymmetrical flow patterns developed in the swirling flow tundish were mainly caused by the swirling flow out of the swirling chamber, partly because of turbulence fluctuations.

Diaz et $a l^{74)}$ in another study concluded that the SLS avoids efficiently the formation of vortexes and recirculating flows either under isothermal and non isothermal conditions. SLS enhances floatation of inclusion and makes the floatation rate less dependent on particle size. The trajectories of inclusions in the water flow are shown in Figs. 18, 19 and 20.

Thus the SLS is a good alternative to substitute for the

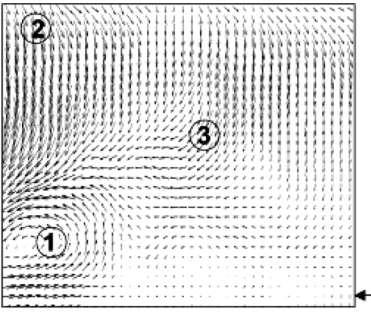

a) $\rightarrow 0.012 \mathrm{~m} / \mathrm{s}$

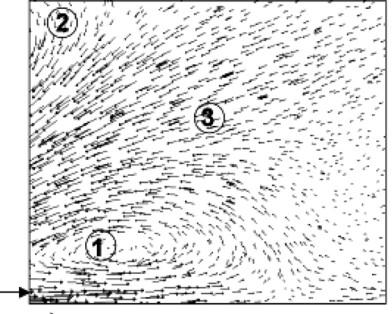

c) $\rightarrow 0.013 \mathrm{~m} / \mathrm{s}$

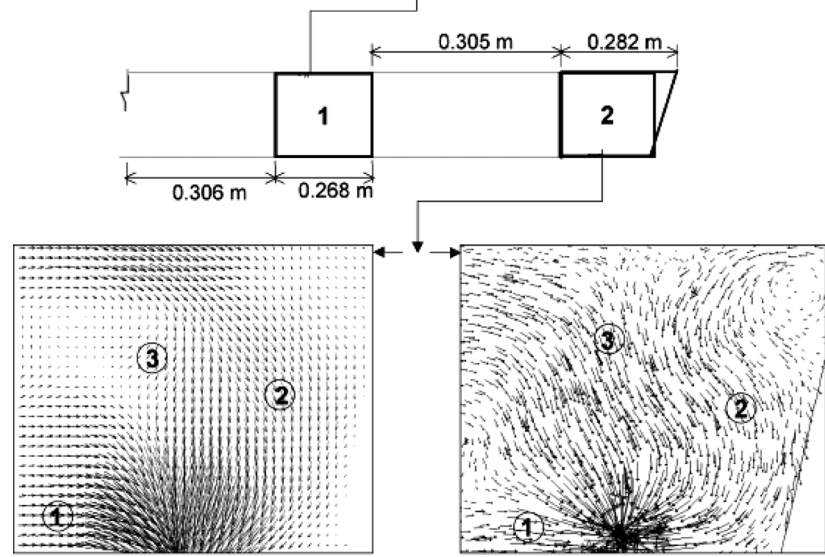

b) $\rightarrow 0.02 \mathrm{~m} / \mathrm{s}$

Fig. 14. Measured and mathematically predicted velocity fields using the RSM model at symmetry longitudinal planes; (a) PIV measurements at plane 1, (b) mathematical predictions at plane 1, (c) PIV measurement at plane 2, and (d) mathematical predictions at plane 2 . 
FCD available in the market. The effect of the swirling ladle shroud (SLS) on fluid flow and mass transfer in a water model of a tundish was studied by Diaz et al. ${ }^{75)} \mathrm{Ex}-$ perimental and mathematical simulations of liquid steel, using the water model, were presented using two kinds of
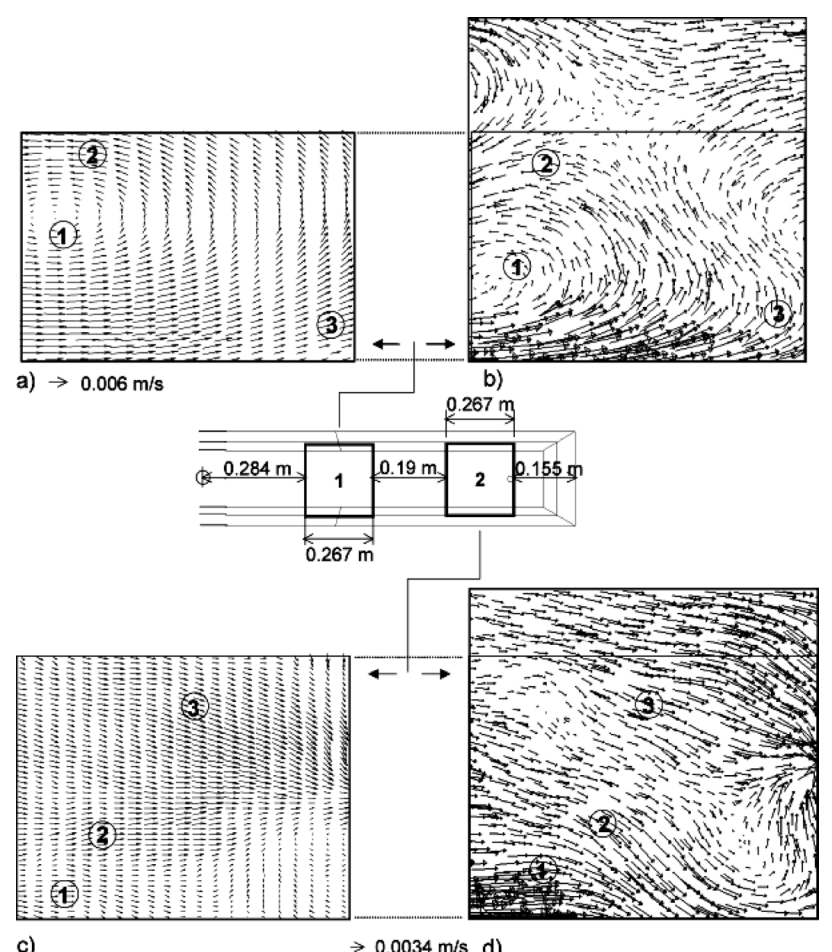

Fig. 15. Measured and mathematically predicted velocity fields (using the RSM model) at symmetrical horizontal planes; (a) PIV measurements at plane 1, (b) mathematical predictions at plane 1, (c) PIV measurements at plane 2, and (d) mathematical predictions at plane 2 .

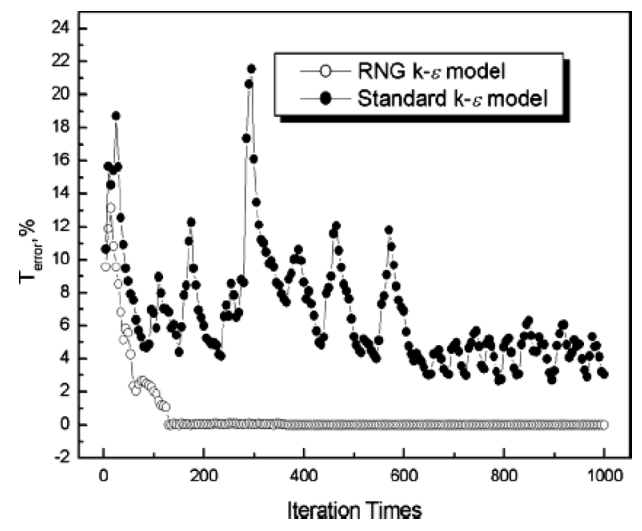

Fig. 16. Variation of total error with iteration number.

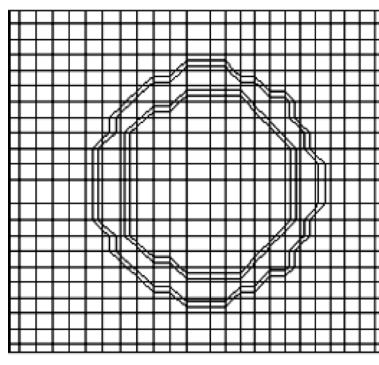

(a)

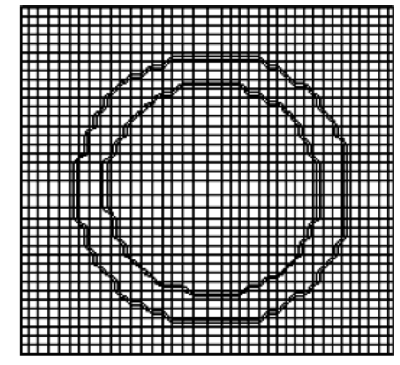

(b)
Fig. 17. Grid systems of $x-y$ plane; (a) sparse grid and (b) dense grid.
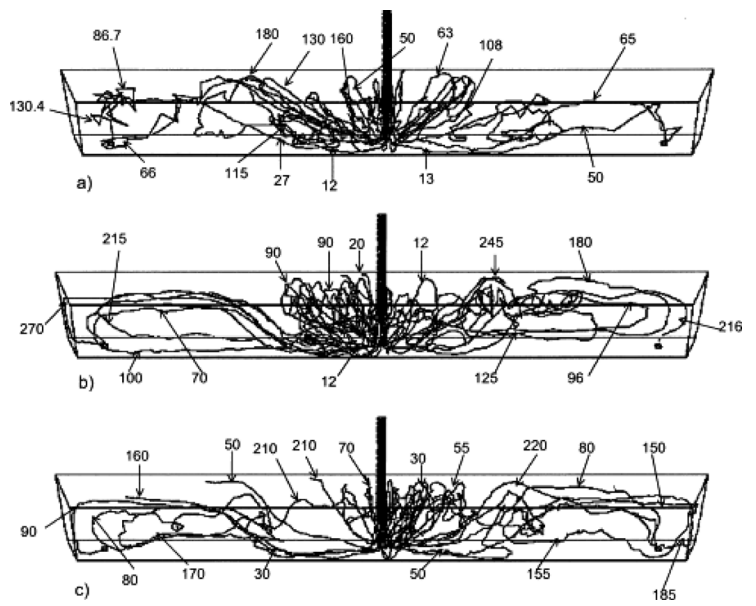

Fig. 18. Trajectories of inclusions in the water flow obtained by mathematical simulation: isometric view using conventional ladle shroud and particles of 20 microns; (a) $27 \mathrm{~s}$ after, (b) $120 \mathrm{~s}$ after, and (c) $240 \mathrm{~s}$ after, the thermal step input.
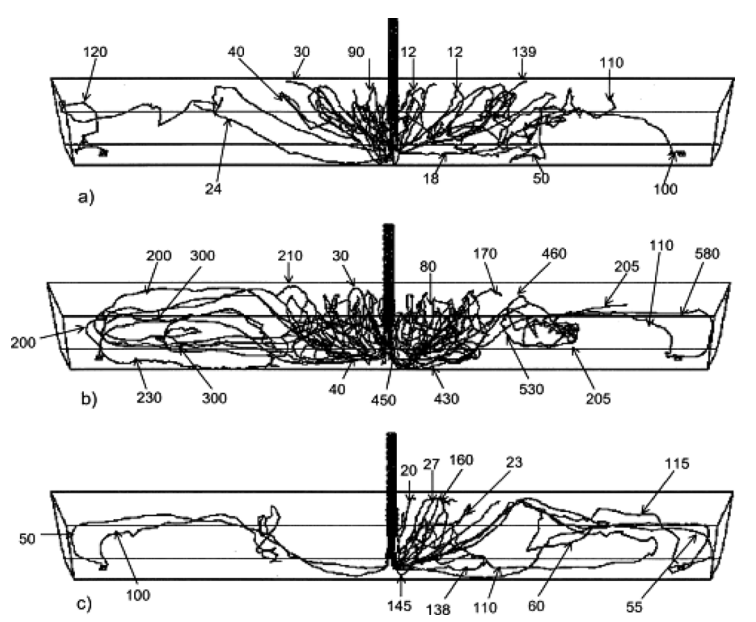

Fig. 19. Trajectories of inclusions within the flowing water, obtained by mathematical simulation. Isometric view using conventional ladle shroud and particles of 100 microns; (a) $27 \mathrm{~s}$ after, (b) $120 \mathrm{~s}$ after, and (c) $240 \mathrm{~s}$ after, the thermal step input.

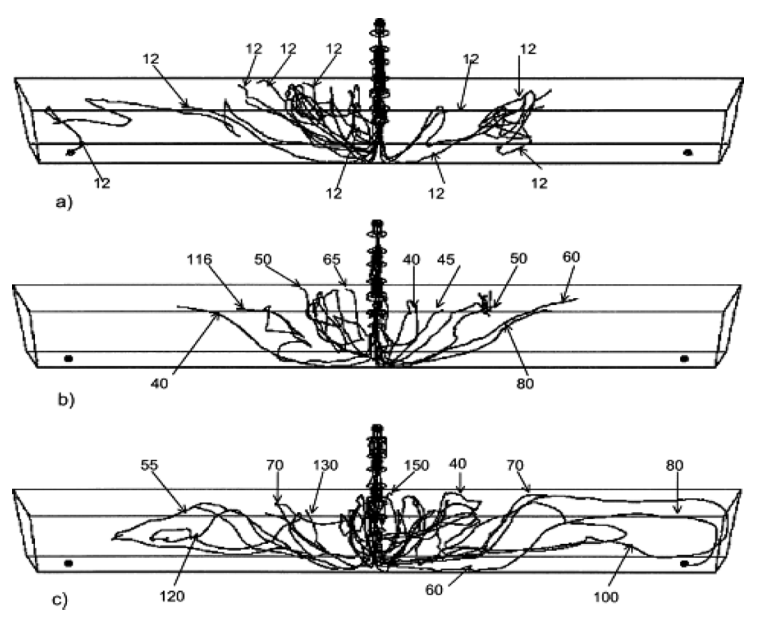

Fig. 20. Trajectories of inclusions in the water flow obtained by mathematical simulation. Isometric view using swirling ladle shroud and particles of 20 microns; (a) $27 \mathrm{~s}$ after, (b) $120 \mathrm{~s}$ after, and (c) $240 \mathrm{~s}$ after, the thermal step input. 
ladle shroud; a conventional ladle shroud and a swirling ladle shroud that dissipates the turbulent kinetic energy by rotational motion of the entry jet in the tundish. They used the $\kappa-\varepsilon, \kappa-\omega$ and the Reynolds stress model (RSM). Tracer dispersion could be accurately predicted by the RSM model while the $\kappa-\varepsilon$ and $\kappa-\omega$ models showed poor predictions. The RSM model also proved to be efficient enough to simulate acceptably well the velocity fields determined experimentally by PIV measurements. All the three turbulence models predicted close results among themselves for RTD curves but the RSM was in best agreement with the experimental minimum residence time.

The SLS may have many advantages but from the flow fields it appears that the flows are highly complex and may cause erosion of refractory inside the shroud which is not economical and detrimental for the steel quality.

After a thorough review on fluid flow and turbulence in tundishes, it is a good idea to discuss non-isothermal melt flows in the tundishes. The non-isothermal nature of melt flows may be due to energy losses that take place from the top surface and through the walls and bottom of the tundish. More importantly, it is also possible that the temperature of the inlet stream into the tundish from the ladle may vary from heat to heat or with teeming time from the same ladle of steel. Thus, there are many situations in actual practise where the temperature of the incoming stream from the ladle is different from that of the molten steel already present in the tundish. It has been shown by Chakraborty and Sahai, ${ }^{76)}$ and by Joo and Guthrie, that the fluid flow patterns developed in such cases are quite different from those obtained under isothermal conditions. Nonisothermal conditions give rise to natural convection and buoyancy driven flows within a tundish that are of a comparable order of magnitude to the inertially-driven components. Thus, it is very useful that water modeling can simulate the non-isothermal aspects of the fluid flow phenomena taking place in a continuous casting tundish. Damle and Sahai $^{77)}$ examined the necessary modeling criteria for a non-isothermal tundish system. For non-isothermal flows, it is necessary to satisfy thermal similarity in addition to the geometric and dynamic similarities. Fluid flows and mixing of molten steel in a twin-slab-strand continuous casting tundish were investigated using a mixing model under non isothermal conditions by Alizadeh et al. ${ }^{78)}$ This model led to a set of ordinary differential equations that were solved with a Runge-Kutta algorithm. Steady state water modelling tests were carried out under non-isothermal conditions. Experimental data obtained from the water model were used to calibrate the mixing model. As a result of the presence of mixed convection phenomena in the non-isothermal tundish, parts of the primary fluid were mixed in with the warm incoming fluid. Due to the density difference between the two fluids, fluid channelling became evident within the tundish. The volume flow rate of the fluid in the channel was found to depend on the ratio of inertial to buoyancy forces inside the tundish. They mentioned that if $\mathrm{Re}_{\mathrm{T}}$ and $\mathrm{Tu}$ between two tundishes are same, (i.e. model and prototype), then the RTD curves would be in absolute accordance with each other. The mixing model results showed that the total mixed flow volume fraction in the non-isothermal tundish was lower than that for an isother- mal one. Sheng and Jonsson ${ }^{79)}$ in MEFOS, Sweden, reported on convection patterns of liquid steel in the continuous casting tundish under non-isothermal conditions. They concluded that the convection pattern is controlled by a combination of natural convection and forced convection i.e. mixed convection. Some researchers at Baosteel ${ }^{80)}$ reported on numerical simulation of coupled fluid flow and heat transfer to enhance their tundish design. They had problems of temperature homogenization in the tundish. However, after doing a thorough investigation of fluid flow, RTD and temperature distributions, under non-isothermal conditions, they were able to solve their problems. Morales et $a l .{ }^{81)}$ reported on buoyancy effects on the melt flow pattern in continuous slab casters. Thermal responses of stepinput temperatures in steel for different flow rates were predicted. The molten steel velocity profiles were determined for these temperature and flow rate variations. The importance of natural flow convection was established through a dimensionless number given by the ratio $G r / R e^{2}$. Buoyancy forces proved to be as important as inertial forces, especially in the extremities of the tundish. The simulations indicated that these forces increase the residence time of the molten steel flowing near the slag layer, allowing more time for the non-metallic inclusions to be captured. They also found that the turbulence inhibiting device helps to redirect the flow towards the top free surface. It was shown that this device helped to decrease turbulence near the entry zone and has a damping effect on the temperature step inputs, allowing for better control of the casting temperature. The traditional flow control devices such as weirs and dams were not as effective as the turbulence inhibitor. Shamik Ray, ${ }^{82)}$ while at the MMPC, did some water modelling work to analyse non-isothermal melt flows. In his water modelling experiments and in his mathematical simulations, it was observed that during step inputs of hot water into a relatively cooler liquid in the tundish, the buoyancy driven flows cause the incoming hotter fluid to move above the remaining colder fluid and to move ahead of it.

\section{Concluding Remarks}

A thorough review of fluid flow and turbulence, physical and mathematical modelling has been carried out. It is seen that much work has been accomplished in the development of mathematical models for studying fluid flow pattern in tundishes, to study the effect of FCD's, to evaluate mean residence time, RTD's and inclusion removal. These advances are due to the increase in computational power and decrease in computational costs. However, slag entrainment, one of the most important issues in continuous casting, has not gained much attention and only a few researchers have reported that in the literature. Also, it is essential to validate these numerical models with actual experiments. The review also shows a recent trend in modelling real life non isothermal conditions. In reality, tundish operations are non-isothermal and there are effects of inlet steel temperature and buoyancy forces. So isothermal modelling predictions may not be correct since tundish flows are very sensitive to even minor fluctuations in steel/slag temperature (i.e. $\sim 5^{\circ} \mathrm{C}$ ). Also, many researchers are now opposed to the use of FCD's and are suggesting to compen- 
sate for their absence by increasing the tundish volume for longer residence tims and better inclusion removal. This practice has already started in Japan a long time ago together with the adoption of plasma heating for maintaining isothermal conditions during casting. Although, DNS seems to be impossible, a few research centres at Japanese Steel Companies are doing it with the aid of parallel processing with over 300 computers working in synergy.

\section{Nomenclature}

Re: Reynolds number

$L$ : Characteristic length, $\mathrm{m}$

$U:$ Average velocity of the fluid, $\mathrm{m} / \mathrm{s}$

$\rho$ : Density of the fluid, $\mathrm{kg} / \mathrm{m}^{3}$

$\sigma:$ Surface tension at any interface

$P$ : Pressure at any point, $\mathrm{Pa}$

$V_{f}$ and $V_{r}$ : Fluid and Stokes rise velocities respectively, $\mathrm{m} / \mathrm{s}$

$\mu$ : Viscosity of the fluid, $\mathrm{kg} / \mathrm{m}-\mathrm{s}$

$\tau$ : Shear stress on the fluid, $\mathrm{N} / \mathrm{m}^{2}$

$v_{x}$ or $u$ : X-component of velocity of the fluid, $\mathrm{m} / \mathrm{s}$

$v_{y}$ or $v: \quad$ Y-component of velocity of the fluid $\mathrm{m} / \mathrm{s}$

$v_{z}$ or $w$ : Z-component of velocity of the fluid $\mathrm{m} / \mathrm{s}$

$T$ : Temperature, $\mathrm{K}$

$t$ : Time, $\mathrm{s}$

$\vec{V}: \quad$ velocity vector, $\mathrm{m} / \mathrm{s}$

$F_{b}$ : Body force, $\mathrm{N}$

$\bar{U}_{i}$ : Reynolds averaged velocity component, $\mathrm{m} / \mathrm{s}$

$U_{i}$ : Instantaneous velocity component, $\mathrm{m} / \mathrm{s}$

$\kappa$ : Kinetic energy of turbulence per unit mass, $\mathrm{m}^{2} / \mathrm{s}^{2}$

$\varepsilon$ : Rate of energy dissipation, $\mathrm{m}^{2} / \mathrm{s}^{3}$

$G_{K}:$ Rate of production of $\mathrm{K}, \mathrm{kg} / \mathrm{m}-\mathrm{s}^{3}$

$C_{1}, C_{2}, C_{\mu}, \sigma_{\kappa}$ and $\sigma_{\varepsilon}$ : Empirical constants

Bo: Bond Number

Tu: Tundish Richardson number

PDE's : Partial differential equations

Subscript

$$
\begin{array}{cl}
m: & \text { Model } \\
p: & \text { Prototype }
\end{array}
$$

\section{REFERENCES}

1) D. Mazumdar and R. I. L. Guthrie: ISIJ Int., 39 (1999), No. 6, 524

2) R. I. L. Guthrie and M. Isac: IISTech Conf., 2003, Indianapolis.

3) R. I. L. Guthrie: Engineering in Process Metallurgy, Oxford University Press, (1992).

4) http://www.answers.com/topic/bond-number

5) S. Tanaka: PhD Thesis, McGill University, (1989).

6) K. Krishnapisharody and G. Irons: ICS 2008, (2008), 367.

7) C. Damle and Y. Sahai: ISIJ Int., 36 (1996), 681.

8) Sahai and Emi: Tundish Technology for Clean Steel Production, World Scientific Publication Co. Pte. Ltd., (2008).

9) A. Ghosh: Secondary Steel Making, Principles and Applications, CRC Press, (2001).

10) S. Lopez-Ramirez, J. de J. Barreto, P. Vite-Martinez, Serrano, J. A. Romero and C. Duran-Valencia: Metall. Mater. Trans. B, 35B (2004), No. 5, 957.

11) A. Vargas-Zamora, R. D. Morales, M. Diaz-Cruz, J. Palafox-Ramos, D. L. Garcia: Int. J. Heat Mass Transfer, 46 (2003), 3029.

12) K. J. Craig, D. J. De Kock, K. W. Makgata and G. J. De Wet: ISIJ Int., 41 (2001), No. 10, 1194.

13) T. Debroy and J. A. Sychterz: Metall. Mater. Trans. B, 16B (1985), 497.

14) B. E Launder and D. B. Splading: Lectures in Mathematical Models of Turbulence, Academic Press, NY, (1972).

15) Q. Hou and Z. Zou: ISIJ Int., 45 (2005), No. 3, 325.

16) P. K. Jha, R. Ranjan, S. S. Mondal and S. K. Dash: Int. J. Numer. Methods Heat Fluid Flow, 13 (2003), No. 8, 964.

17) H.-J. Odenthal, R. Bolling and H. Pfeifer: Steel Res. Int., 74 (2003), No. $1,44$.

18) H.-J. Odenthal, R. Bolling, H. Pfeifer, J.-F. Holzhauser and F.-J. Wahlers: Steel Res., 72 (2001), Nos. 11-12, 466.

19) H.-J. Odenthal, H. Pfeifer and M. Klaas: Steel Res., 71 (2000), Nos. 6-7, 210.

20) T.-H. Shih, W. W. Liou, A. Shabbir, Z. Yang and J. Zhu: A New $\kappa-\varepsilon$ Eddy Viscosity Model for High Reynolds Number Turbulent Flows-Model Development and Validation, NASA TM 106721, (1994).

21) O. J. Ilegbusi: ISIJ Int., 34 (1994), No. 9, 732.

22) P. K. Jha and S. K. Dash: Int. J. Numer. Methods Heat Fluid Flow, 12 (2002), No. 5, 560.

23) A. Robert and D. Mazumdar: Steel Res., 72 (2001), No. 3, 97.

24) H. Solhed, L. Jonsson and P. Jonsson: Metall. Mater. Trans. B., 33B (2002), No. 2, 173.

25) H. Solhed and L. Jonsson: Scand. J. Metall., 32 (2003), No. 1, 15.

26) A. Kumar, S. C. Koria and D. Mazumdar: ISIJ Int., 44 (2004), No. 8, 1334.

27) G. Solorio-Diaz, R. D. Morales and A. Ramos-Banderas: Int. J. Heat Mass Transfer, 48 (2005), No. 17, 3574.

28) G. Solorio-Diaz, R. D. Morales, J. Palafox-Ramos and A. RamosBanderas: ISIJ Int., 45 (2005), No. 8, 1129.

29) S. V. Patankar and D. B. Spalding: Int. J. Heat Mass Transfer, 15 (1972), 1787.

30) H. K. Versteeg and W. Malalasekera: An Introduction to Computational Fluid Dynamics: The Finite Volume Method, McGraw-Hill, Loughborough University, (1995).

31) C.-M. Fan and W.-S. Hwang: ISIJ Int., 40 (2000), No. 11, 1105.

32) H.-J. Odenthal, H. Pfeifer and M. Klaas: Steel Res., 71 (2000), No. 6-7, 210.

33) D. Y. Sheng and L. Jonsson: Metall. Mater. Trans. B, 31B (2008), 867.

34) J. H. Ahn, J.-E. Lee and J. K. Yoon: 2nd Int. Conf. on Processing Materials for Properties, TMS, Warrendale, PA, (2000).

35) S. K. Sinha, K. M. Godiwalla, D. K. Shaw and C. S. Sivaramakrishnan: Scand. J. Metall., 30 (2001), 103.

36) Y. Sahai: Modelling of Melt Flow in Continuous Casting Tundishes, The Brimacombe Memorial Symposium, 631.

37) S. P. Ferro, R. J. Principe and M. B. Goldschmit: Metall. Mater. Trans. B, 32B (2001), 1185.

38) P. Gardin, M. Brunet, J. F. Domgin and K. Pericelous: Appl. Math. Model., 26 (2002), 323.

39) P. K. Jha and S. K. Dash: Int. J. Numer. Methods Heat Fluid Flow, 14 (2004), No. 8, 953.

40) R. Pradeshi, S. Basak, A. K. Singh, B. Basu, V. Mahashabde, S. K. Roy and S. Kumar: ISIJ Int., 44 (2004), No. 9, 1534.

41) A. V. Zamora, R. D. Morales, M. Diaz-Cruz, J. Palafox-Ramos and J. De J. Barreto-Sandoval: Metall. Mater. Trans. B, 35B (2004), 247.

42) A. Ramos-Banderas, R. Sanchez-Perez, R. D. Morales, J. PalafoxRamos, L. Demedices-Gracia and M. Diaz-Cruz: Metall. Mater. Trans. B, 35B (2004), 449.

43) A. Tripathi and S. K. Ajmani: ISIJ Int., 45 (2005), No. 11, 1616.

44) M. J. Cho and I. C. Kim: ISIJ Int., 46 (2006), No. 10, 1416.

45) X. Huang and B. G. Thomas: Metall. Mater. Trans. B, 27B (1996), 617.

46) N. Alkishriwi, M. Meinke and W. Schroder: Aerodynamics Institute, RWTH Aachen, Germany, (2007), 396.

47) O. Davila, R. D. Morales and L. Gracia-Demedices: Metall. Mater. Trans. B, 37B (2006), 71.

48) R. Sankanarayan and R. I. L. Guthrie: Ironmaking Steelmaking, 29 (2002), 147.

49) R. Sankanarayan and R. I. L. Guthrie: ISS Steelmaking Conf., Vol. 75, (1992), 655.

50) P. Vayrynen, S. Vapalahti, S. Louhenkilpi, M. Clark and T. Wagner: STEEL SIM 2007, Austria.

51) M. Zorzut, F. Vecchiet, N. Kapaj and A. Paderno: BHM, 152. Jg. (2007), Heft 11.

52) I. Hamil and T. Lucas: Proc. of TMS Annual Meeting on Fluid Flow 
Phenomena in Metals Processing, TMS, Warrendale, PA, (1999), 279.

53) S. Joo and R. I. L. Guthrie: Can. Metall. Q., 30 (1991), 261.

54) R. Schwarze, F. Obermeier, J. Hantusch, A. Franke and D. Janke: Steel Res., 72 (2001), Nos. 5-6, 215.

55) J. Hulstrung, M. Zeimes, A. Au, W. Oppermann and G. Redusch: Steel Res., 76 (2005), No. 1, 59

56) L. Zhang, S. Taniguchi and K. Cai: Metall. Mater. Trans. B, 31B (2000), 253

57) M. Javurek, B. Kaufmann, G. Zuba and P. Gittler: Steel Res., 73 (2002), No. 5, 186

58) B. G. Thomas and Hua Bai: 18th Process Technology Division Conf. Proc., Vol. 18, Iron and Steel Society, Warrendale, PA, (2001), 895.

59) J. P. Rogler, L. J. Heaslip and M. Mehrvar: Can. Metall. Q., 43 (2004), No. 3, 407

60) A. V. Nguyen, J. Ralston and H. J. Schulze: Int. J. Miner. Process., 53 (1998), 225.

61) A. V. Kuklev et al:: Metallurgist, 48 (2004), Nos. 3-4, 000.

62) J. P. Rogler, L. J. Heaslip and M. Mehrvar: Can. Metall. Q., 44 (2005), No. 3, 357.

$63)$ S. Lopez-Ramirez, R. D. Morales and J. A. Romero Serrano: Numerical Heat Transfer, Part A, 37 (2000), 69.

64) R. Schwarze, F. Obermeier and D. Janke: Modelling and Simulation in Material Science and Engineering, 9 (2001), 279.

65) R. D. Morales, M. Diaz-Cruz, J. Palafox-Ramos, S. L. Ramirez and J. De J. Barreto Sandoval: Steel Res., 72 (2001), No. 1, 11.

66) Y. Bao, W. Jang, B. Xu and H. Zhang: J. Univ. Science and Technology, Beijing, 9 (2002), No. 1, 13.
67) J. Palafox-Ramos, J. De J. Barreto, S. L. Ramirez and R. D. Morales: Ironmaking Steelmaking, 28 (2001), No. 2, 101.

68) R. D. Morales, S. L. Ramirez, J. Palafox-Ramos and D. Zacharias: Ironmaking Steelmaking, 28 (2001), No. 1, 33.

69) K. H. Tacke and J. C. Ludwig: Steel Res., 58 (1987), 262.

70) A. K. Sinha: PhD Thesis, The Ohio State University, USA, (1990).

71) H. Nakajima, F. Sebo, S. Tanaka, L. Dumitru, D. J. Harris and R. I. L. Guthrie: Steelmaking Conf. Proc., ISS-AIME, (1986), 69, 705.

72) G. S. Diaz, R. D. Morales, J. Palafox-Ramos, L. G. Demedices and A. R. Banderas: ISIJ Int., 44 (2004), No. 6, 1024.

73) Q. Hou and Z. Zou: ISIJ Int., 45 (2005), No. 3, 325.

74) S. Diaz, R. D. Morales, J. Palafox-Ramos and A. R. Banderas: ISIJ Int., 45 (2005), No. 8, 1129.

$75)$ G. S. Diaz, R. D. Morales and A. R. Banderas: Int. J. Heat Mass Transfer, 48 (2005), 3574.

76) S. Chakraborty and Y. Sahai: Metall. Trans., 23B (1992), 152.

77) C. Damle and Y. Sahai: ISIJ Int., 36 (1996), 681.

78) M. Alizadeh, H. Edris and A. Shafyei: J. Iron Steel Res. Int., 15 (2008), No. 2, 7. 22.

79) D. Y. Sheng and L. Jonsson: Technical Report by MEFOS, S 97125, Lulea, Sweden.

80) Baosteel Group Co.: Company Publications, Technical Report, 200512-29.

81) R. D. Morales et al.: Modelling Simul. Mater. Sci. Eng., 8 (2000), 781.

82) S. K. Ray: PhD Thesis, McGill University, Montreal, Canada, (2009). 